Review Article

\title{
In Situ Real-Time TEM Reveals Growth, Transformation and Function in One-Dimensional Nanoscale Materials: From a Nanotechnology Perspective
}

\author{
Nikolay Petkov \\ Electron Microscopy and Analysis Facility, Materials Chemistry and Analysis Group, Tyndall National Institute, Cork, Ireland \\ Correspondence should be addressed to Nikolay Petkov; nikolay.petkov@tyndall.ie
}

Received 14 November 2012; Accepted 28 November 2012

Academic Editors: E. Cattaruzza, C.-L. Hsu, and W. Lu

Copyright (c) 2013 Nikolay Petkov. This is an open access article distributed under the Creative Commons Attribution License, which permits unrestricted use, distribution, and reproduction in any medium, provided the original work is properly cited.

This paper summarises recent developments in in situ TEM instrumentation and operation conditions. The focus of the discussion is on demonstrating how improved understanding of fundamental physical phenomena associated with nanowire or nanotube materials, revealed by following transformations in real time and high resolution, can assist the engineering of emerging electronic and optoelectronic devices. Special attention is given to $\mathrm{Si}, \mathrm{Ge}$, and compound semiconductor nanowires and carbon nanotubes (CNTs) as one of the most promising building blocks for devices inspired by nanotechnology.

\section{Introduction}

Every aspect of basic nanoscale science as well as commercial production of nanotechnologies is dependent upon the capacity of instruments and methodologies to measure, sense, fabricate, and manipulate matter at the nanoscale. Microscopy has the advantage over other characterisation techniques (e.g., bulk spectroscopy or electrical testing) in that it is descriptive, producing images of objects that are directly related to their structure, morphology, and composition, and hence it directly uncovers spatial heterogeneities. Nowadays, lattice resolution images of crystalline materials are acquired routinely by high resolution transmission electron microscopy (TEM) using conventional TEM optics. In the last few years, the state-of-the-art has become the sub-Angstroms resolution imaging and analysis, achieved through the use of image or probe aberration-corrected TEM instruments [1]. Using the ability of forming an extremely small and intense electron probe within aberration-corrected scanning transmission electron microscope (STEM), quantifiable images of atomic columns and indeed atom-by-atom visualisation have been demonstrated [2].

Observing processes "on site" as they are occurring and under changing external stimuli is the paramount goal of in situ time resolved techniques. Various in situ and operandi techniques have emerged and are gaining importance in different areas of science and engineering. In the field of nanoscience and nanotechnology, there are only a handful of techniques that can merge extreme spatial resolution with the possibility of in situ real-time detection. Among them, in situ electron microscopy is probably the most versatile and mature technique, and it has been a topic of separate workshops, dedicated books, and extended reviews [3-12]. It emerged in the 1960s, driven by the technology needs to examine stability and radiation damage of materials for aviation, nuclear reactors, and space exploration. In the past, it was not rare that dynamical changes were observed as unwanted outcomes of examinations when highly energetic $(1 \mathrm{MeV})$ electron beams were used, hence supplying important information on radiation damage in materials [13]. Nowadays, almost every aspect of nanoscale discovery and nanotechnology-driven processes can benefit from the in situ TEM technique. Due to its extreme spatial resolution, it is not surprising that in situ TEM is responsible for the discovery of completely new transformation phenomena in systems such as clusters of atoms, nanoparticles, nanotubes, or nanowires that are difficult to examine by other in situ characterisation techniques.

One-dimensional materials such as nanowires and nanotubes are intrinsically miniaturised; the magnitude of the 
material is confined to a length of less than $100 \mathrm{~nm}$ in two dimensions. This makes them ideally suited for experiments within a transmission electron microscope, minimizing the need for elaborated sample thinning. Undoubtedly, this is not the only reason to examine nanowires by electron microscopy. Nanowires and nanotubes possess properties governed by their miniaturised dimensions and large surfaceto-bulk ratio that afford them a fundamental scientific interest. Technologically, nanowires, and in particular semiconductor nanowires, have a vast range of potential applications, including electronic (logic devices, diodes), photonic (laser, photodetector), biological (sensors, drug delivery), energy (batteries, solar cells, thermoelectric generators), and magnetic (spintronic, memory) devices [34-37]. On the other hand, metallic nanowires and nanotubes can both act as interconnects for the transport of charge carriers as well as active device elements, for example, as biochemical or gas sensors [38]. The emergence of nanotechnology and the development of these fascinating applications rely on the rational control over the electronic and optical properties of nanowires and the chemical synthesis (including the adjustment of the morphology, size, and material composition in various nanowire structures), all of which can only be observed by electron microscopy due to the finite size of nanowires.

Electron microscopy investigations accompany almost every publication concerning nanowire or nanotube growth, assembly and integration into functioning devices, hence the application of electron microscopy to the study of onedimensional nanoscale materials has been reviewed [39]. One can identify three levels of information supplied by electron microscopy: initial studies of the nanowire or nanotube dimensions, overall composition, and crystallinity (single, polycrystalline, or amorphous). These are directly obtained by conventional TEM imaging (normally through massthickness or diffraction contrast imaging under bright field conditions), energy dispersive X-ray (EDX) analysis, and selected area electron diffraction (SAED). This type of information, if statistically relevant, is used to provide important feedback that is used for rational design of growth conditions or postgrowth processing steps for a particular application. The second type of information is more detailed and concerns in-depth characterisation of nanowire growth directions, surface faceting and passivation, intermixing and impurity concentrations, and last but not least various types of crystallographic defects that may exist in the nanowire or nanotube structure. Such characterisation is normally associated with more complex nanowire or nanotube topologies such as coreshell nanowires, axially heterostructured nanowires, and surface functionalised nanowires. Such type of information is provided by phase contrast imaging, weak beam dark field (WBDF) imaging, nanobeam or convergent beam electron diffraction (CBED), and examinations of different nanowire tilt angles and nanowire cross-sections. When complimented by high resolution elemental mapping using fine scanning electron probes combined with EDX or electron energy loss spectroscopy (EELS), almost full description of nanowire structure and composition is obtained. This type of information is important for devising nanowire functionality, providing an understanding of the material properties that affect the electronic or optical response of nanowire-fabricated devices. The third level of information that can be obtained by electron microscopy studies is related to direct advancement of our understanding of the surface energetics and the formation mechanisms of one-dimensional materials, as well as to their interaction and response to external stimuli that are of technological relevance. In this case, it is required to answer two fundamental questions. (i) How can atoms or other building blocks be rationally assembled into structures with nanosized diameters but much longer lengths or, in other words, how do nanowires grow? (ii) What are the intrinsic properties of these structures, such as strength, conductivity, melting point, and phase diagrams and how do these properties depend on diameter, structure and composition under exposure to varying external stimuli?

These are the main questions which this paper addresses by presenting a comprehensive summary of recent advances in the in situ TEM instrumentation and associated operation as well as by highlighting studies that brought progress in recognizing the major key factors at play when growing, transforming, or influencing the function of one-dimensional structures. The transformations triggered by the electron beam radiation, although intriguing due to the ability to precisely modify materials structure, demonstrating single atom transformations in some occasions [40], are excluded from the discussion. Special attention is given to $\mathrm{Si}, \mathrm{Ge}$, and compound semiconductor nanowires and carbon nanotubes (CNTs) as one of the most promising building blocks for devices inspired by nanotechnology. The focus of the paper is on in situ TEM studies of dynamic transformations that are directly associated with the future manufacture of nanowireor nanotube-based electronic devices such as FETs, phase change memory, Li-ion batteries, and NEMs.

\section{In Situ TEM Instrumentation and Operation}

Modern in situ electron microscopy is only made possible by the recent advances in the electron microscopy instrumentation. Recent years have seen not only the development of dedicated designs and modifications of the electron microscope columns but also the immense improvement of auxiliary attachments such as dedicated holders, stages, and reaction cells for various in situ processes. Nowadays, instruments and attachments for in situ electron microscopy are not only laboratory-made systems but are also commercially available. In this section, a review of the modern instrumentation for in situ TEM is given, including the development of dedicated environmental TEM systems, various TEM stages for applying thermal, electrical, and mechanical stimuli within the TEM. The operation of the aberration-corrected optics for in situ electron microscopy, and ultrafast in situ electron microscopy instrumentation will also be briefly discussed.

In general, specimen and microscope requirements for in situ TEM or STEM imaging are not much different from those of conventional TEM or STEM operation. The major difference is in the ability of applying external conditions and influences (stimuli) inducing changes in the specimen and some method of recording and analyzing the resulting 
dynamic responses. At present, most TEM systems are equipped with a charge-coupled device (CCD) able to operate at very fast rates as well as with television- (TV-) rate cameras. These can record images down to about several tens of milliseconds. Any cause of a substantial drift, bending, or tilting of the specimen during recording images is a major limiting factor for high resolution dynamic observations. This will make subsequent interpretation, extracting any timedependent differences in the sample evolution being very limited and prone to errors. Therefore, it is important to optimise the stability of the system (sample, holder, and electron optics) to the best of our abilities. Obviously, these limitations will be minimised if we have the possibility to record images at very fast acquisition rates as within the ultrafast electron microscope (UFEM). Experiments on picosecond, or even femtosecond time scales, have been carried out by applying pulsed electron beam synchronised with transformations in the sample triggered by ultrafast laser beams. This advance is based on fundamentally different concept of timed coherent single-electron packets, or electron pulses, which are liberated with femtosecond durations delivered by lasers coupled to the electron source. When the emission of the picosecond laser coupled to the electron source is clocked with the one used to stimulate the sample, dynamic transformations with extreme temporal resolution, for example, ultrafast TEM, can be obtained. The operation and examples of using ultrafast electron microscopy, including the benefits of using aberration-corrected electron optics, for studying nano- and picosecond dynamics of various processes have already been reviewed and described in specialised books [12, 41, 42]. These will not be covered in the following sections of this paper. It is important to note that this technique opens an exciting opportunity of observing transformations and reactions that happen at femtosecond time scale which when coupled to the existing extreme spatial resolution of aberration-corrected instruments will transform in situ electron microscopy into dynamic molecular/atomic observatory, visualising atoms, and molecules reacting.

2.1. The Environmental In Situ TEM. Ever since the discovery of the electron microscope in 1930s, a goal has been to image samples in different environments (gas or liquid, and not exclusively in high vacuum) as easy as with an optical microscope but with much higher resolving power [43]. Conventional electron microscopes require high vacuum $\left(10^{-6}\right.$ Torr or higher) within the specimen chamber, both to allow the operation of the electron source and to minimise scattering other than from the sample. On the other hand, many processes with fundamental importance in the material science, such as, for example, crystal growth or catalytic processes, are taking part at atmospheric or higher gas pressures. A standard characterisation protocol for studying kinetics of such processes by TEM is to sample specimens, ex situ, at different reaction times. Unfortunately, detailed dynamic information of the key transformations during, for example, vapour-liquid solid growth or gas phase catalytic reactions is only possible to obtain by performing in situ reactions within the TEM. This led to the development of a dedicated electron microscope and a corresponding technique called environmental electron microscopy $[5,14]$. Typically, this technique is used to describe the operation of the electron microscope at reduced vacuum by allowing different reaction gases including "wet" inert gases to be introduced in the sample chamber. Herein, the discussion will focus on the development of the environmental TEM (E-TEM). On the other hand, the environmental scanning electron microscopy (E-SEM) has, nowadays, become almost conventional mode of operation, and this capability is readily available on many SEM systems provided by all major electron microscopy suppliers.

In order to introduce different gas environments within the sample compartment of the TEM, respectively, STEM, there are generally two ways to accommodate the excessive gas pressures: (i) to modify the entire electron column (the electron optics and the vacuum system) and (ii) to use specially designed sample cells where the excessive gas pressure is only contained in a very close vicinity of the examination volume. The first approach adds a differential pumping system to the electron column, which makes the system much more expensive, with higher maintenance costs compared to the regular TEM systems. The advantage is the possibility to obtain high resolution imaging, sample tilting, and heating. The second approach is much more price efficient and simple and requires minimal or no modification of the TEM column itself. Obvious disadvantage is that the region around the sample has increased thickness due to the introduction of additional layers to sustain the gas pressure around it, which limits the obtainable resolution during the imaging. The first practical example of E-TEM instrument for high resolution imaging has been developed by Boyes and Gai in 1997, based on modification of the electron column of a Philips CM30 high resolution TEM [14, 44]. The design of the system (see schematics in Figure 1(a)) included radial holes in the objective pole pieces for the first stage of the differential pumping and the number of apertures separating the regular sample chamber from the rest of the column by differential pumping lines. The differential pumping was achieved by connecting molecular drag pumps (MDPs) or turbo molecular pumps (TMPs) between the apertures. Since then, FEI [45], Hitachi [15], and JEOL [46] all developed dedicated E-TEM instruments, which are generally all based on this approach. Most importantly, they are now commercially available and can be assembled on demand. Although being much more expensive than the conventional TEMs, these instruments are becoming increasingly accessible to researchers and are gaining popularity. Specifically, when the E-TEM module is coupled to an aberration-corrected optics, systems that can be custom made by the aforementioned suppliers, then the instruments become extremely powerful in observing transformations at extremely high resolution, under high temperature and varying gas environment. The advantage of having aberration-corrected optics is in the possibility to obtain atomic resolution images in a single exposure [47]. This is extremely important as it is not possible to record a systematic trough-focus series of images for subsequent exit wave function reconstruction (a popular method for obtaining quantitative structural information with improved resolution) while the sample 


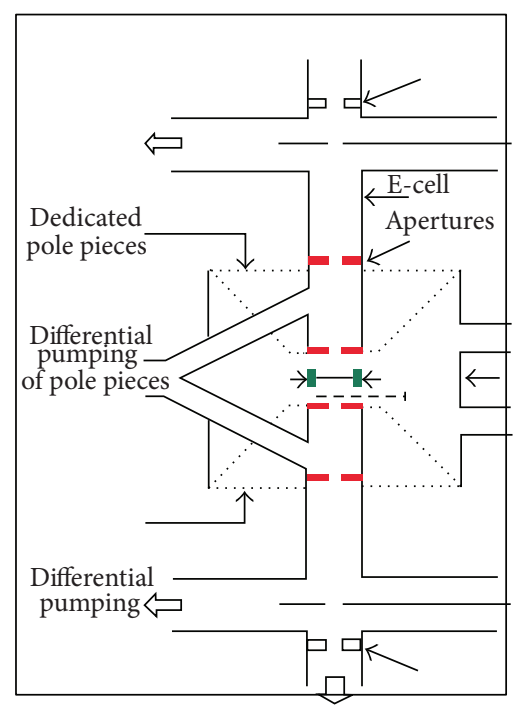

(a)

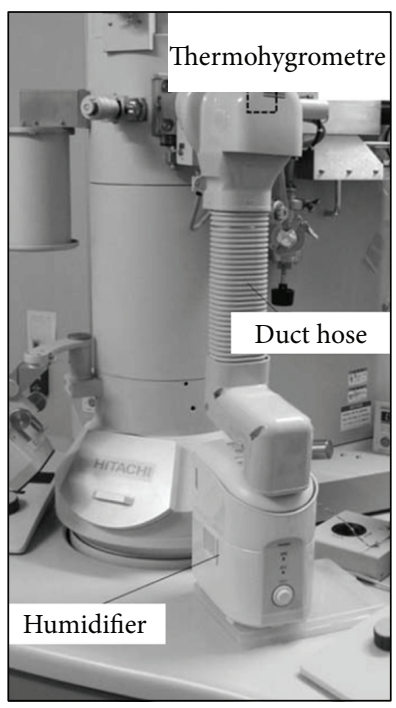

(b)

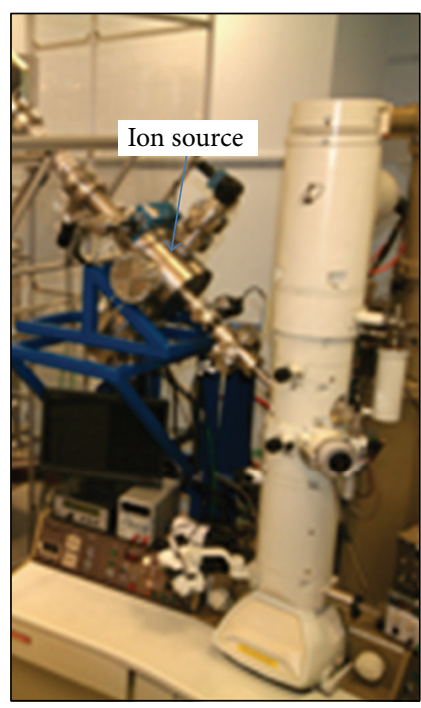

(c)

Figure 1: (a) Schematics of E-TEM set-up featuring differential pumping, dedicated apertures, and pole piece, adapted from [14]. (b) Photograph of a gas cell designed to deliver humidified gases directly through the specimen arm, adapted from [15]. (c) Photograph of ion-irradiation column attached to the TEM for in situ measurements, adapted from [16].

is continuously changing. Still not commercially available are the instruments featuring environmental in situ low energy electron microscopy (LEEM) capabilities. In general, LEEM instruments operate at UHV and are used to examine surface properties of crystalline materials and formation of terraces and very thin layers [48]. Dedicated instruments assembled in laboratories can be interfaced with a gas supply systems to study dynamics of formation of thin layers, surface reconstruction, and other surface-supported materials such as metal clusters and islands.

Recently, a method to introduce gas around the sample via gas injection nozzle fitted on a sample heating holder which is part of the specimen arm has been developed (see Figure 1(b)). The other parts of the electron column (e.g., specimen chamber, objective lens pole pieces, and the vacuum system) remained unchanged. This relatively less complicated and inexpensive approach allows imaging of gas in situ reactions at lattice resolution at room temperature. Similar gas injection systems (GISs) are widely used for deposition of metals and oxides using the power of secondary electrons (SE) emitted by scanning electron or ion beams to decompose different gas precursors [49].

\subsection{Dedicated In Situ Stages, Holders, and Reaction Cells. The} possibility to transform materials by using external influences within the TEM using a modified specimen arm or by introduction of custom-made stages or reaction cells has received major attention in the recent year [7-9]. There are many different types of TEM specimen arms and holders capable of heating or cooling, straining, lasing, evaporating/depositing materials or applying current/voltage, and so forth, that are available commercially or can be fabricated without great difficulty in the laboratory. All of these devices do not require major modification of the electron optics nor the vacuum system in contrast to the E-TEM instruments. A comprehensive description of the modes of operation and possible working conditions of these devices is given in the following sections.

2.2.1. Heating Stages. Many reactions and transformations such as crystal growth, recrystallization, and phase transitions in solids take part at elevated temperatures. In situ heating stages for TEM are normally integrated as part of the specimen arm and use precise Joule heating and temperature dissipation to obtain tunable and stable sample temperatures. Figure 2(a) depicts a Gatan single tilt heating stage that can accommodate a $3 \mathrm{~mm}$ disk specimen and can reach $1200^{\circ} \mathrm{C}$ when using a Tantalum furnace [17]. When it is the aim to perform high resolution TEM imaging at elevated temperatures, it is imperative to obtain high $\left(<0.1^{\circ} \mathrm{C} / \mathrm{min}\right)$ temperature stability and low sample drift. This is difficult to achieve with a large sample stage that has a very large thermal mass and, respectively, slow temperature response. To address this issue, microfabricated heating stages were developed and are commercially available by Protochips Inc [50] and DENs solutions [18] (Figure 2(b)). These use integrated circuitry to produce localised resistive heating directly on the sample support. Such stages show much lower thermal mass and very high thermal stability that allow high resolution imaging at elevated temperatures. Additionally, this type of stage offers disposable design of the heating elements, for example, the integrated circuit platform (chip) that reduces contamination and ensures compatibility practically with any microscope.

2.2.2. Electrical Holders. The electrical transport measurements of individual or arrays of one-dimensional nanostructures are commonly assessed by using electrical probing stations after developing contacts by optical or electron beam lithography [51]. The probing stations are equipped with optical microscopes that are normally only used to 


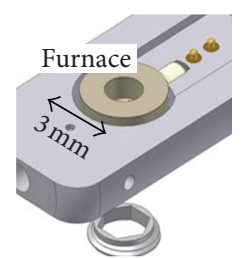

(a)

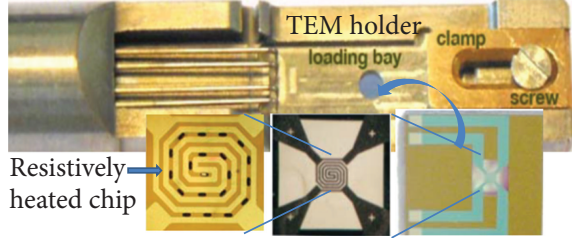

(b)

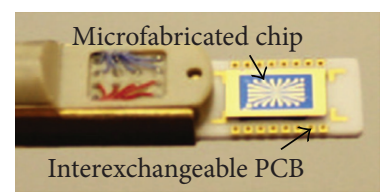

(c)

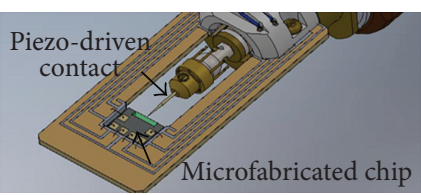

(d)

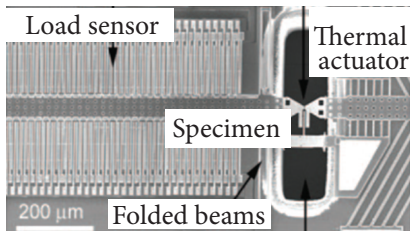

(e)

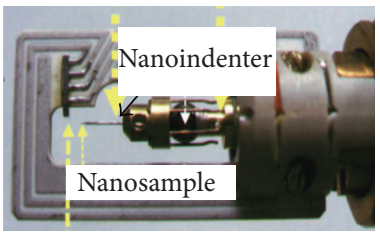

(f)

Figure 2: (a) Cartoon of a heating stage that accommodates full-size TEM grids commercially available from Gatan [17]. (b) Photographs of microfabricated heating stage commercially available from DENS solution [18]. (c) Photograph of custom-made TEM electrical holder using microfabricated circuitry, adapted from [19]. (d) Cartoon of TEM holder with nanocontacting and microfabricated chip capabilities, commercially available from Nanofactory Instruments AB [20]. (e) Custom-made microfabricated MEMs chip that can be interfaced with TEM specimen arm, adapted from [19]. (f) TEM holder with nanoindenter capabilities, commercially available from Nanofactory Instruments $\mathrm{AB}[20]$.

ease the navigation of the electrical probes and for rough identification of the structures for analysis. On the other hand, scanning probe microscopy (SPM) methods such as scanning tunnelling microscopy (STM), scanning resistance microscopy (SRM), or scanning capacitance microscopy (SCM) were used for mapping electronic and dopant properties of individual devices on a chip level. Nowadays, electrical probing stages for electrical contacting within the TEM have been developed and are commercially available from several suppliers such as Nanofactory Instruments $\mathrm{AB}$, Hummingbird, and Gatan (Figure 2). There are generally two types of holders: (i) with integrated circuitry for contacting microfabricated chips and (ii) with piezo-driven nanoprobe contact. The first approach uses prefabricated circuitry developed by lithography on silicon chips containing thin silicon nitride membranes (see Figure 2(c)) [22, 52, 53]. The nanowires or nanotubes are dispersed on electron transparent membrane and connected to the prefabricated circuitry by direct writing metal interconnects by electron or ion beam-induced deposition within an SEM or FIB. The chips are finally fitted within the TEM specimen arm, and the contacting is achieved via wire bonding. The stages that use conductive nanoprobes are brought into contact to one end of the nanowires or nanotubes directly by precise piezodriven manipulator (see Figures 2(d) and 2(f)). The other end of the nanostructure is contacted (glued by conductive paste) to unmovable metal wire. Practically, the first approach involves many more steps and large micro-fabrication effort. The second approach is relatively more straightforward but requires a quite large investment for purchasing a dedicated piezo-driven stage. Notably, both types of stages can be used not only for electrical testing but also for selective heating of nanoscale materials by Joel heating [54-57]. Alternatively, when inserted in an E-TEM, such stages are well suited to grow nanowires [31]. Recently, Nanofactory Instruments and our group have started developing an approach converging piezo-driven nanocontacting capabilities with circuitry for microfabricated chips (see Figure 2(d)). The key advantage of using this type of holder is that nanowire-based devices obtained by top-down processing can be electrically tested using more than two terminals. The most challenging part of this approach is to obtain electron transparent regions of interest but not to compromise the function of the devices.

2.2.3. Mechanical Stages. Nanoindentation and atomic force microscopy techniques are commonly used to investigate mechanical properties of nanoscale materials such as very thin layers of nanowires and nanotubes as well as nanoparticles [58]. These techniques use probes (fine tips) interacting with the sample surface. Unfortunately, it is not rare that specimen/tip interactions can only be inferred but not visualised independently. Similarly to the electrical probing within the TEM, holders capable of applying mechanical stress while observing structural transformations using the electron beam have been developed. These are generally based on using two approaches: (i) microfabricated microelectromechanical systems (MEMSs) that are fitted within a TEM specimen arm [59-61] and (ii) dedicated nanoindentation stages encompassing piezo-driven probes integrated with the TEM specimen arm [33, 62-64]. One of the first examples of MEMS-based nanoscale mechanical testing system (n-MTS) for TEM has been custom made using state-of-the-art microfabrication (Figure 2(e)) [19]. In this system, load is applied using a thermal actuator on one side of the freestanding specimen and is measured on the other side, using a differential capacitive load sensor based on interdigitated electrodes. The nanowire specimen is mounted between the actuator and load sensor shuttles. The nanowire specimens are either fabricated by top-down manufacturing as suspended bridges or can be assembled from grown nanowire materials. The 
latter technique requires methods for precise placing of the grown nanowires between the interdigitated electrodes. As for the electrical probing, piezo-driven nanoindenter or AFM TEM holders have frequently been applied to probe the mechanical properties of grown nanowires only (Figure 2(f)). It is worth to mention that MEMs platforms as well as nanomanipulators that can be observed under the SEM while applying electrical or mechanical stimuli exist as well. Their fabrication and integration within the SEM chamber is to some degree less demanding due to the larger available volume around the specimen.

2.2.4. Liquid Cells for TEM. The desire to observe liquid samples in the electron microscope dates back to its discovery [65]. For decades, this problem was unsolved due to the difficulties in sustaining large but still electron transparent volumes of a liquid sample within the electron column. Within the last decade, this limitation has been overcome by designing what we now call "liquid cells" for high resolution electron microscopy [66]. This attracted immediate attention not only from the bio- and life-sciences users of the electron microscopy technique, but it also opened vast possibilities for the materials science community. The recent advances in in situ TEM using liquid cells have been recent reviewed [10]. The liquid cell design is remarkably different to the environmental in situ TEM in that it has the possibility to sustain a thin and stable liquid layer. The realisation of the liquid cell concept for high resolution TEM is a result of the recent rapid advances in micro- and nanofabrication and in particular fabrication of thin membranes and microfluidic channels. It represents a hermetically sealed enclosure that constrains the liquid into a layer less than a few micrometres thick between thin electron transparent windows. The very thin electron transparent windows made of silicon nitride or silica are fabricated and hermetically sealed using CMOS-based manufacturing (see Figure 3(a)) [21, 67, 68]. Very recently, an alternative approach has been demonstrated using singlelayered graphene sheets to enclose small volumes (pockets) of liquid (Figures 3(b) and 3(c)) [23]. This methodology has the advantage of lattice resolution imaging due to the extremely small electron scattering volume in comparison to silicon nitride-based liquid cells. The advantage of using microfabricated membrane liquid cells is in that they can be assembled in a way that they contain microfluidic channels delivering controlled flow of liquid. Additionally, in such cells, it has been demonstrated that electrodes can be incorporated so that electrochemical growth can be triggered and followed in situ [66]. Nevertheless, processes such as, for example, growth by solvothermal synthesis or directed self-assembly have not yet been examined by using the liquid cell TEM approach. These will require novel design of the liquid cell by introducing, for example, localised heating elements and mixing valves within the microfluidic channels and electron transparent windows that can sustain high vapour pressure.

2.2.5. TEM Instruments with Lasers and Ion Beam Irradiation Capabilities. The idea of introducing additional beam capabilities for modifying (milling, etching, doping, or laser heating) the specimen while imaging with the electron beam has led to the development of complex systems which can generally be described as multibeam systems. The laser or ion sources are normally easier to integrate as a part of the electron column of the SEM due to the larger specimen chamber. Nowadays, all major producers of electron microscopes have systems named Dual Beam (FEI), CrossBeam (Carl Zeiss), and MultiBeam (JEOL) that have SEM and focus ion beam (FIB) capabilities on a single platform. Ion beam capabilities for TEM are only possible on custom-built systems, available in only several laboratories in the UK, France, Japan, and the United States. A specially designed deflector system is used to direct the ion flux onto the sample surface (Figure 1(c)) [16]. Such facilities are normally used to generate mechanistic data on ion beam irradiation processes in materials used in nuclear reactors. Relatively unexplored is the field of ion beam irradiation in nanoscale materials studied by integrated in situ TEM/ion beam systems. Similarly, laser sources are integrated into an electron microscope and can be used to generate heat or trigger reactions within the specimen. Such systems are not commercially available yet. Normally, an existing TEM column is modified to accommodate laser beam facilities. As mentioned previously, the laser system can be interfaced with the specimen compartment of the TEM column and/or it can be coupled to the electron source. The first laser allows for triggering a reaction or applying certain stimuli into the sample, while the second is used to control the emission of the electron source. An alternative and probably more cost-efficient way to introduce laser beam capabilities in the TEM is to use inexpensive optical fibres or microfabricated light sources and interface them with the TEM specimen arm. Recently, Nanofactory Instruments $A B$ has announced a TEM specimen arm which integrates existing nanocontacting capabilities with an optical fibre [20]. This set-up can potentially be used to study transformations under laser annealing or ablation, measure the black body radiation of a specimen and hence its temperature, or perform cathodoluminescence experiments in the TEM. When near-filed optical probe is coupled to the manipulator within the TEM experiments such as near-field laser ablation or even near-filed optical microscopy and spectroscopy within the TEM can be envisioned.

\section{Nanowire and Nanotubes Growth, Processing, and Function Observed by In Situ TEM}

3.1. Nucleation and Growth of Nanowires and Nanotubes. Nanowires and nanotubes can be grown by a variety of synthetic methods. The most popular are (i) electrochemical deposition within nanoporous templates, normally used to grow metallic nanowires, (ii) various forms of metal-catalysed CVD growth used to grow semiconductor nanowires as well as carbon nanotubes (CNTs), and (iii) solvothermal growth of semiconductor and oxide nanowires and nanotubes [69]. Details regarding these methods can be found in the other reviews and references therein. Most importantly, only a small fraction of the developed growth 


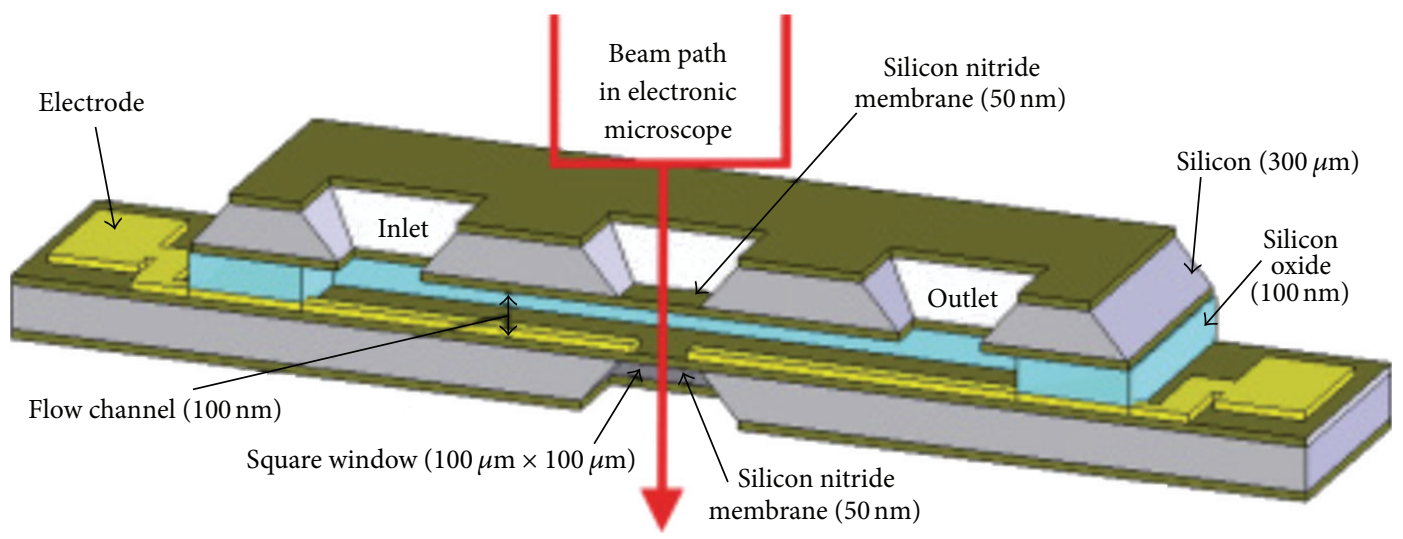

(a)

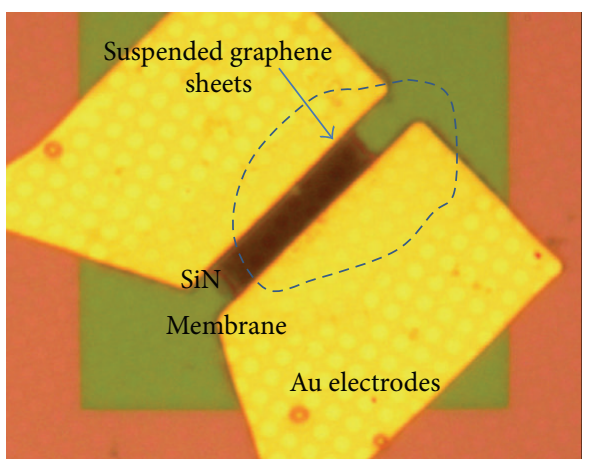

(b)

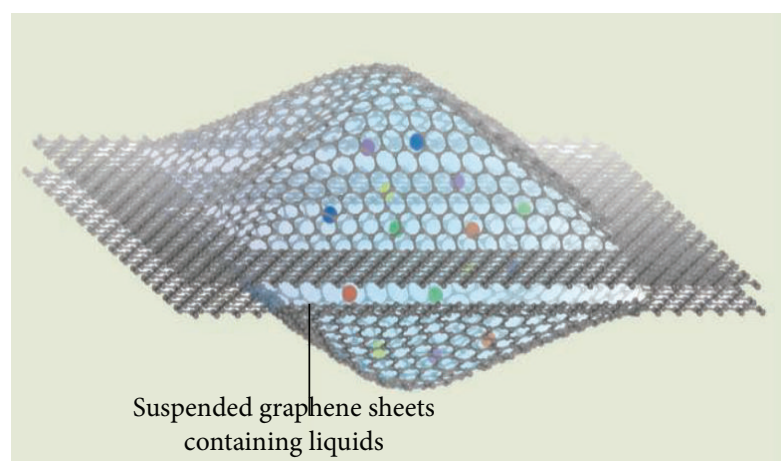

(c)

FIGURE 3: (a) Schematics of liquid TEM cell design featuring microfabricated circuitry and microfluidic delivery, adapted from [21]. (b) Optical micrograph of SiN membrane cells containing microfabricated circuitry and suspended graphene sheets, adapted from [22]. (c) Cartoon of graphene sheets encompassing miniscule volume of liquid, adapted from [23].

process has found its route to detailed mechanistic examination by the in situ TEM technique. For example, in situ electrochemical growth of metals within the TEM has been demonstrated but not in the form of templated growth of nanowires. In situ reactions in liquid phase forming metal clusters and nanoparticles have been observed in the TEM but only at room temperature and not under solvo-thermal conditions. Other techniques such as atomic layer deposition (ALD) or laser-assisted growth and laser-induced crystallisation are not yet examined by in situ TEM. Probably, the most studied growth mode by in situ TEM is the VLS growth technique and closely associated metal-catalysed growth of CNTs. These techniques received major attention not only because of their technological importance but also because some of their scientific fundamentals that are not fully understood can be best described with unprecedented detail by performing in situ TEM measurements. The instrumentation that is best suitable for observing such processes is based on the ETEM instrument where gas-solid and gas-liquid interactions are followed at elevated temperatures and under specific gas environments. The in situ E-TEM of nanowire and nanotube growth has already been reviewed in the past [5]; herein, we focus on more recent studies by summarising and contrasting general growth trends and crystallisation dynamics for both CNTs and semiconductor nanowires.
The large number of possible variables associated with the metal-catalysed growth of CNTs and semiconductor nanowires makes it difficult to isolate the individual role of the various synthesis parameters during their formation. Ex situ postgrowth TEM studies are indispensable in analysing CNTs and semiconductor nanowires; such studies accompany almost every paper on synthesis of CNTs and semiconductor nanowires and commonly demonstrate that the catalyst particle dictates the morphology and diameter of the grown structures. However, such postgrowth characterisation is static and can be ambiguous due to the changes during cooling and transfer. There are also key questions, such as how do nanotubes and nanowires nucleate and whether the catalyst is liquid or solid, what is the actual dynamics of the transformations, and do these transformations obey the steps that are commonly associated with commonly accepted growth mechanisms. In the later stages of the growth process, the measurement of growth rates under various conditions allows the rate-limiting steps to be determined, further confirming the growth model at play. Finally, the dynamic data obtained can be used to reevaluate the general formulation used to describe the growth thermodynamics and kinetics by accounting for phenomenological differences due to the nanoscale dimensions. 
3.1.1. Growth of CNTs Observed by In Situ TEM. The first high resolution in situ E-TEM study of CNTs involved CVD-type growth catalysed by $\mathrm{Ni}$ nanoparticles and precursor gases such as $\mathrm{C}_{2} \mathrm{H}_{2}$ or $\mathrm{CH}_{4}$ at gas pressures of about $10^{-6} \mathrm{mbar}$ with temperatures set to $600^{\circ} \mathrm{C}$ or higher [70]. It is important to stress that the conditions used were similar to those used in normal metal-catalysed growth of CNTs so that it was possible to draw realistic conclusions about the key parameters at play. This process has been studied on several occasions in the following years in more detail $[24,71,72]$. The general agreement is that in the initial stages of CNT growth, the $\mathrm{Ni}$ nanoclusters tend to reform their shape forming a highly elongated shape (Figure 4(a)). Moreover, a different mechanism of formation of the hollow and bamboo-type multiwalled CNTs has been directly observed. For example, when being in the initial stages of nucleation, the Ni nanoparticle elongation is followed by an abrupt contraction, then conformal carbon overcoating is formed, hence building the characteristic bamboo-like overlaps. In comparison, when a tangential extrusion of graphene layers without carbon coating of the elongated catalyst particle is in place, the result is the formation of a hollow multiwalled CNT. One more mechanistic finding obtained is that the metal particle remains crystalline in the course of the CNT growth. This suggests that the formation mechanism in the early stages follows steps such as precursor adsorption and decomposition, surface diffusion, and CNT-wall nucleation instead of a precipitation reaction as seen during the VLS growth (see what follows). Further growth is achieved by carbon diffusion at the metal nanoparticle/carbon multistep interface. Another aspect of the growth, which is commonly observed, is that the linear growth rate is intercepted when a kink or other defect is introduced in the CNT structure. With regard to the measured rate of growth of the multiwalled CNTs, it varies significantly between 0.34 and $25.2 \mathrm{~nm} / \mathrm{s}$, and it is largely dependent on the temperature used and type of CNTs formed, for example, multiwalled, bamboo-like, and so forth.

\subsubsection{Metal-Catalysed Growth of Semiconductor Nanowires} Observed by In Situ TEM. The metal-catalysed growth of semiconductor nanowires such as $\mathrm{Si}, \mathrm{Ge}$, and others has been conducted in the E-TEM instrument by dosing gases such as $\mathrm{SiH}_{4}$ and $\mathrm{Ge}_{2} \mathrm{H}_{6}$, at pressures in the $10^{-6}$ Torr range. In comparison to the ones used for CNTs growth, these precursors are highly reactive at elevated temperatures (highly explosive as well when in contact with air) and require very clean ultrahigh vacuum (UHV) environment. Such conditions can be achieved within a UHV TEM, although it is rather challenging. Another major difference between the metal-catalysed growth of CNTs and the one conducted for semiconductor nanowires is that the latter can be grown epitaxially on the corresponding substrates and under correct conditions.

The first examples we consider here are from the recent years, where Si nanowire growth in E-TEM is achieved after dosing $\mathrm{Si}_{2} \mathrm{H}_{6}$ over Au nanoparticles supported on oxide-free 110 oriented Si substrates [73] or silica-coated TEM grids [25]. The first stages of the reaction involve $\mathrm{Au}-\mathrm{Si}$ eutectic alloying and formation of a liquid seed droplet, in a full agreement
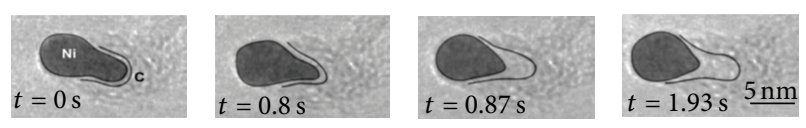

(a)
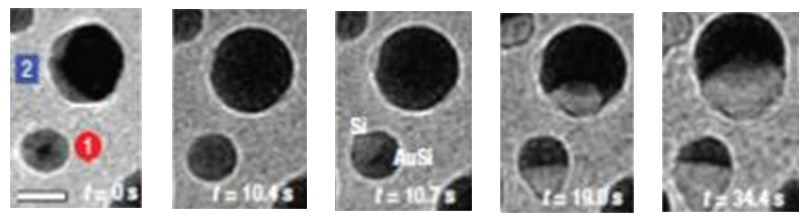

(b)

FIGURE 4: Sequence of TEM images demonstrating time-dependent transformations during the nucleation of multiwalled CNTs using $\mathrm{Ni}$ catalysts ((a), adapted from [24]) and Si nanowires using Au nanoparticles ((b), adapted from [25]).

with the VLS model. It has been shown that $\mathrm{Si}$ nucleation occurs burst-like, by growing into the $\mathrm{Au}-\mathrm{Si}$ eutectic droplet, with smaller catalyst particles nucleating Si first (Figure 4(b)). In earlier publications, similar conditions were used, focusing on understanding the influence of the changing size, shape, and possible diffusion of the Au seeds during the growth of Si nanowires [74]. Initially, the surface of the wafer bearing the Au catalyst was investigated under UHV conditions at $600^{\circ} \mathrm{C}$, showing that Au nanoparticles dewet the surface forming nanoparticle seeds which grow in size with the time due to Oswald ripening. Similarly on the silica surface, the catalyst droplets undergo significant reshaping owing to their high density and coalescence. Remarkably, using the extreme surface analytical capabilities of the low-energy electron microscope (LEEM), it was found that between the forming Au droplets on the $\mathrm{Si}$ surface, there is a monolayer of atomic gold. This layer kept its existence during subsequent Si nanowire growth. It has been observed that the growth rate (about $15 \mathrm{~nm} / \mathrm{s}$ ) is almost independent on the nanowire diameter in the range of 50-150 $\mathrm{nm}$ diameters. Moreover, the Au seeds change their shape and size during growth, resulting in conical Si nanowires with no Au seeds on their tips or wires with changing diameters and even such forming a sawtoothlike nanowire surface [75]. The migration of Au during growth at $600^{\circ} \mathrm{C}$ is driven by surface tension and the desire of the Au droplets to minimise their excess of surface energy. This process is not only shaping the Au liquid droplet, respectively, Si nanowire growth, but also contaminates Si nanowire surface with electron acceptor traps [26], that would have profound effect over the electrical transport properties of the devices based on Si nanowires. The contamination of Si nanowires with single atoms of Au has been recently observed ex situ by aberration-corrected STEM [76].

In situ TEM has also been applied to study semiconductor nanowire growth by other modes (variations of the VLS) by observing gas-solid interactions. In this case, the growth is conducted at a temperature below the formation of eutectic liquid alloy droplet, keeping the metal catalyst solid, hence modifying the growth process to vapour-solid-solid (VSS). 
The exact state of the metal catalyst in this process has been a matter of debate for many years, before first in situ TEM observations of $\mathrm{Au} / \mathrm{Ge}$ nanowire systems were accomplished [26]. The reactions were performed on clean (111) $\mathrm{Si}$ substrates at $10^{-5}$ Torr of $\mathrm{Ge}_{2} \mathrm{H}_{6}$ at temperatures varying between $250^{\circ} \mathrm{C}$ and $435^{\circ} \mathrm{C}$, below and above the eutectic temperature of $\mathrm{Au} / \mathrm{Ge}$, respectively. In these experiments, both liquid Au droplets below the eutectic temperature and solid Au seeds above the eutectic temperature were observed. It was found that the formation and the metal catalyst state depend primarily on the temperature history and precursor pressure where both liquid and solid metal seeds can coexist on different wires at the same temperature (Figure 5). Most importantly, Ge nanowires continue to grow when the catalysts are solidified at a growth rate of $1.9 \times 10^{-2} \mathrm{~nm} / \mathrm{s}$ much lower than when grown with a liquid metal on the tip. The fact that below the eutectic temperature liquid metal droplets are seen at high Ge precursor pressure is another important observation that compliments the understanding of the VLS model. It has been suggested that supersaturating the Au catalyst with Ge is responsible for stabilisation of the liquid droplet below the eutectic temperature. Nanoscale effects have been used to describe the changes associated with the $\mathrm{Au}-\mathrm{Ge}$ phase diagram when nucleating Ge growth (Figure 5). The experiments used grown Ge nanowires with Au nanoparticles at their tips heated in the temperature range $300-500^{\circ} \mathrm{C}$ and no gas flux containing Ge precursor $[27,77]$. By measuring the Au-Ge liquid drop volume at increasing temperature, it was possible to quantify the alloy composition as a function of temperature for drops associated with $\mathrm{Ge}$ nanowires with different diameters. Thus, new liquidus lines in the Au-Ge phase diagram were obtained which demonstrate an increase in the suppression of the temperature of formation of the corresponding liquid alloys as a function of the nanowire size (Figure 5). Recently, the Au-catalysed Ge nanowire growth using $\mathrm{Ge}_{2} \mathrm{H}_{6}$ on $\mathrm{SiO}_{2}$ under subeutectic isothermal conditions $\left(260^{\circ} \mathrm{C}\right)$ has also been studied [78]. The results reveal that kinetic effects should also be considered carefully as they can cause local variations in the composition, for example, Ge supersaturation to form $\mathrm{Au}-\mathrm{Ge}$ liquid nanoparticles below the eutectic and not necessarily only the nanoscale effects. The liquid Au-Ge is explained by using formulations derived from bulk thermodynamics and kinetics theory, clarifying why such a metastable phase can either stay liquid or nucleate solid Ge depending on the Ge supersaturation. Briefly, with a continuously rising Ge supersaturation, the kinetic barrier for Ge crystal nucleation falls until Ge step (lattice plane) occurs [79]. This latest study presents for the first time lattice-resolved imaging of crystalline planes during nanowire growth.

The examples of in situ TEM observations shown previously demonstrate that metal-catalysed growth of semiconductor nanowires is much more complex than what is generally described by the VLS or other associated growth models. Thermodynamic as well as kinetic growth effects at the nanoscale need to be considered resulting in the reevaluation of the conventional binary phase diagrams. The major observations obtained by the in situ TEM measurements reviewed
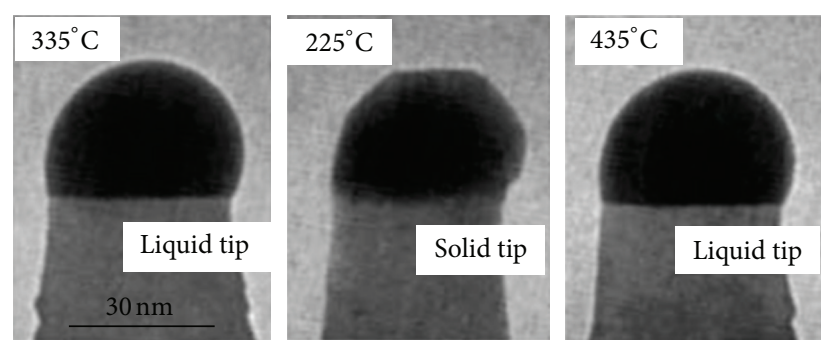

(A)

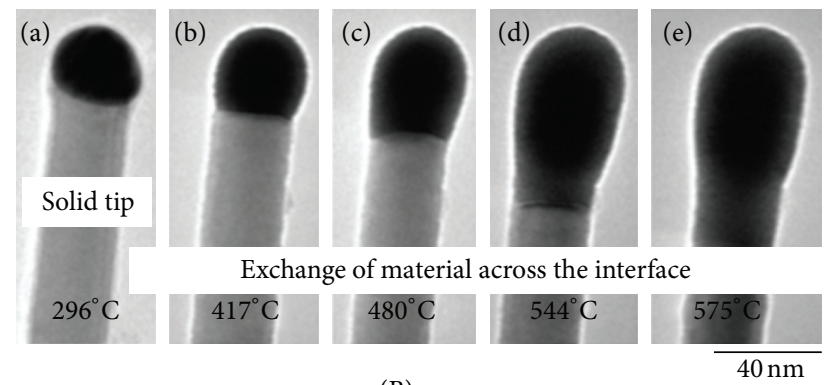

(B)

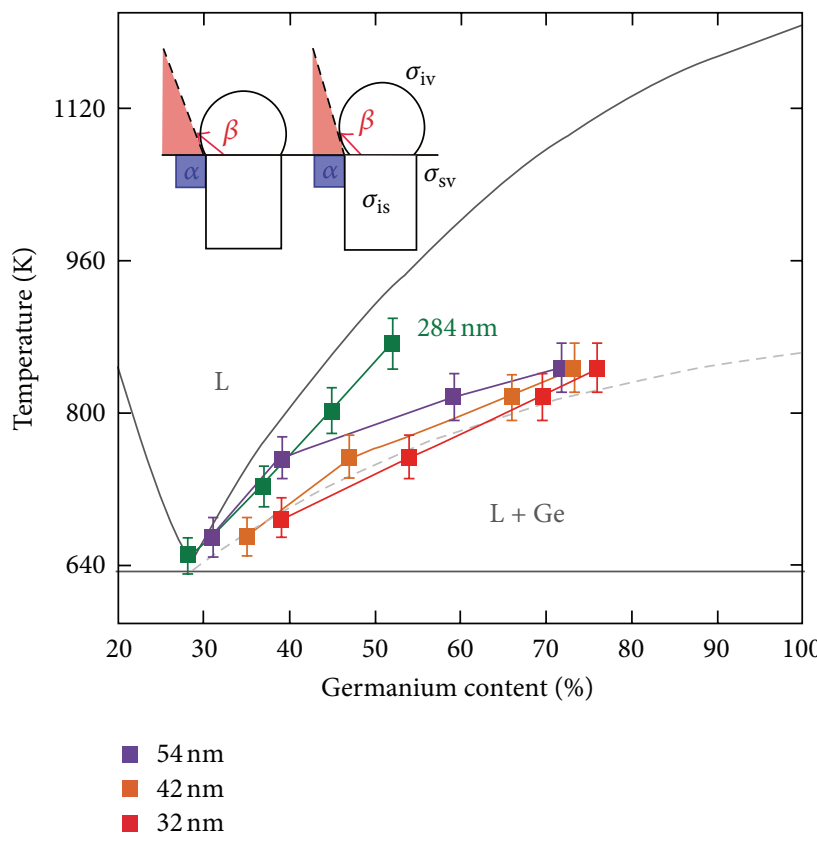

FIGURE 5: Sequence of TEM images showing Au-Ge NW tip at different temperatures and the corresponding transformations. (A) During NW growth at constant digermane pressure (adapted from [26]); (B) under vacuum annealing (adapted from [27]). (C) Au-Ge binary alloy phase diagram outlining new liquidus lines for NWs with different diameters, respectively (adapted from [27]).

previously are (i) the liquid alloy droplet is changing in size, shape, and equilibrium concentration during the nanowire growth, while the conventional VLS growth model does not account for this, (ii) nanowires should form highly faceted surfaces (including sawtooth-like) ruled by the energetics of the crystal growth and minimizing the associated surface energies; this effect is smoothened due to noncatalysed growth that is normally also present, and (iii) migration of 
the alloyed droplet and its constant change, including the formation of metastable phases, can be a limiting factor in the nanowire growth, and it is not accounted for in the conventional VLS model.

3.1.3. Other Nanowire Growth Processes Observed by In Situ TEM. In addition to metal-catalysed growth of semiconductor nanowires, there exist a range of other bottom-up routes to produce semiconductor nanowires. Their manufacturing and applicability in device fabrication has been recently reviewed [36]. These routes, which do not invoke the VLS growth mechanism, include oriented attachment, metal organic vapour phase epitaxy, molecular beam epitaxy (MBE), soft templating, dislocation driven unidirectional growth, and crystal habit modification. Hence, any of those techniques have yet not been followed in such a detail as the VLS growth mode. Particularly attractive is the possibility to grow nanowires of $\mathrm{Si}, \mathrm{Ge}$, and III-Vs by seedless methods. Technologically, Au, which is the most common metal used to grow such nanowires, is inherently incompatible with semiconductor device manufacturing. Au has a high diffusivity in $\mathrm{Si}$ and also acts as a deep level acceptor and thus has detrimental effects on device performance $[80,81]$. In order to avoid contamination of the semiconductor nanowires during growth, our group and others have devised synthetic routes based on using specially designed precursors, solvents, and growth conditions for seedless growth [82, 83]. By using ex situ methodology, we were able to identify some key growth parameters that indicated possible formation mechanisms. The suggested mechanisms (still not conclusive) include existence of melted Ge nanocrystals that nucleate the growth similarly to the VLS model but without forming an eutectic alloy. Another possible route, probably taking place at the same time, is based on oriented attachment of Ge nanocrystals. Recently, a valuable methodology based on using liquid cells for in situ TEM was applied to follow formation of $\mathrm{Pt}_{3} \mathrm{Fe}$ nanorods and extended $\mathrm{Au}$ crystals by oriented attachment of nanocrystals [84, 85]. For the first time, direction-specific interaction of the nanocrystals was observed at lattice resolution, demonstrating motions and rearrangement of the lattice planes to accommodate precise attachment of the nanocrystals at room temperature. Several stages of the growth process were identified: (i) initial nanoparticle formation, (ii) oriented attachment into nanoparticle chains, and (iii) transformation into single crystalline rods. The growth of the initial nanocrystals was initiated by the electron beam reducing the $\mathrm{Pt}$ and $\mathrm{Fe}$ precursors at room temperature. Some of the initial crystals grew by monomer attachment; others undergo coalescence until they reached a critical diameter where extended onedimensional structures started to form. The extreme spatial resolution achieved during imaging allowed identification of further strengthening of the nanoparticle chains taking place with different rates; for example, initial necks between nanoparticles transformed slower than at the later stages of nanorod strengthening process. These first mechanistic data sets of nanocrystal attachment and growth delivered important information for metallic one-dimensional systems.
Similarly, the seedless growth of Si or Ge would be possible to follow if a specially designed in situ TEM liquid cell capable of performing reactions at elevated temperatures and under a fluidic flow can be assembled. Such type of liquid cell and imaging methodology will benefit the understanding of the growth mechanism for semiconductor and oxide structures, such as, for example, $\mathrm{TiO}_{2}$ nanowires and nanotubes, nanosized zeolites, and metal and oxide nanoparticles, that are normally obtained by solvo-thermal growth.

\subsection{In Situ TEM Studies of Nanowire Transformations under} External Influences. In the next section, in situ TEM studies of nanowire and nanotube transformations under external stimuli which led to improved understanding of processes of fundamental and nanotechnological importance are reviewed. These include transformations such as changes between different phases including amorphous to crystalline transformations in nanowires, defect formation and evolution, electrical contacting, and mechanical manipulation of nanowires. The focus of the discussion will be on explaining how improved understanding of fundamental phenomena during such transformations can assist the engineering of devices such as nanowire-based field effect transistors (FETs), phase change memory devices, nanobatteries, and others.

3.2.1. Formation of Nanowire Silicides and Germanides Observed by In Situ TEM. The ability to observe nanowire or nanotube transformation processes that result in formation of nanoscale metal to semiconductor contacts is technologically important due to the necessity to form reliable and low contact resistance devices [86]. Ex situ TEM methods (postfactum) can be used to identify single crystalline silicides or germanides as a result of interactions of metals such as $\mathrm{Ni}$, $\mathrm{Pt}, \mathrm{Co}$, and others with $\mathrm{Si}$ and Ge nanowires after annealing (ref). Such contacts normally display ohmic and low resistivity performance. The nanowire-based devices such as multigate FETs will strongly benefit from the possibility to control the interface between the contacts and the thin body semiconductor channel as well as the formation of ultrashort channel devices $[87,88]$. The most straightforward route to follow the recrystallisation processes during metal/nanowire interactions is by using the in situ TEM heating holders that are loaded with silicon nitride electron transparent grids. The grids are covered with nanowires, sputtered with the metal, or a mixture of metal and semiconductor nanowires are directly dispersed onto the grids [89-91]. After locating a point of contact between the nanowire and the metal, the temperature of the holder is elevated to a point where crystallisation of a metal silicide takes place. A technologically more relevant approach uses nanowires that are contacted by lithography defined metal contact lines directly on silicon nitride membranes $[28,29]$. Based on such reactions, the kinetics of the growth of a particular silicide phase advancing along the axial direction of the nanowires can be followed (Figure 6). The identification of the obtained phases and the interface between the silicide and the nanowires is achieved normally after the formation by using high resolution imaging. The majority of in situ TEM studies are on Ni silicide formation, 

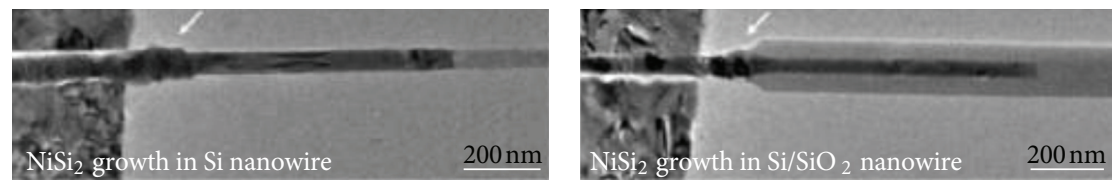

(a)
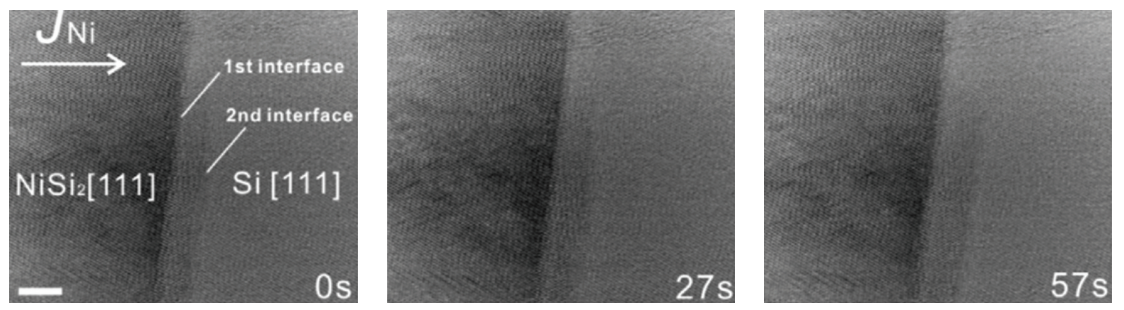

(b)

Figure 6: (a) In situ growth of $\mathrm{NiSi}_{2}$ from $\mathrm{Ni}$ pads along $\mathrm{Si}$ and core-shell $\mathrm{Si} / \mathrm{SiO}_{2}$ nanowires (adapted from [28]); (b) in situ lattice resolution TEM image sequence of a propagating $\mathrm{NiSi}_{2}-\mathrm{Si} \mathrm{NW}$ interface at $360^{\circ} \mathrm{C}$ (adapted from [29]).

thus the kinetics of important silicide such as $\mathrm{NiSi}_{2}$ and $\mathrm{NiSi}$ can be followed. One can expect that before $\mathrm{Ni} / \mathrm{Si}$ growth interactions are observed, the nanowire surface oxide needs to be fully removed. Surprisingly, nanowire Ni silicides were formed with the surface oxide still in place [89]. In this case, it has been observed that the nano-Ni silicide growth starts from the opposite tip rather than from the Ni/Si point of contact. It has been argued that the reason for this is that $\mathrm{Ni}$ atoms diffuse interstitially in $\mathrm{Si}$ and supersaturate at the tip rather than in the contact point where there is limited interaction area due to the existence of the native oxide. The progressing NiSi phase showed an atomically flat interface with a nearly linear growth rate of about $0.11 \mathrm{~nm} / \mathrm{s}$. In a more recent study, it has been confirmed that the growth mechanism is indeed via $\mathrm{Ni}$ diffusion in $\mathrm{Si}$ rather than through the surface oxide and it stops when the $\mathrm{Ni}$ supply is consumed [90]. In any case, epitaxial relations, for example, $\mathrm{Si}[1-10] / / \mathrm{NiSi}[1-12]$ and $\mathrm{Si}(111) / / \mathrm{NiSi}(31-1)$, have been determined for the NiSi phase by SAED studies and lattice resolution images after the reactions.

$\mathrm{NiSi}_{2}$ phase in contact with the $\mathrm{Si}$ nanowires has also been observed $[28,29,91]$, surprisingly in the same temperature interval but only when large supply of $\mathrm{Ni}$ is present. Energetically more favourable is the formation of the NiSi phase as the first principle calculations showed that the formation energy of $\mathrm{NiSi}$ is $\sim-0.488 \mathrm{eV} /$ molecule, and in comparison $\mathrm{NiSi}_{2}$ is $-0.314 \mathrm{eV} /$ molecule. On the other hand, the lattice mismatch between the (111)/(111) set of planes of $\mathrm{NiSi}_{2}$ and $\mathrm{Si}$ is very small in comparison to the mismatch of the (131)/(111) set of planes of NiSi and Si. Hence, arguably, this is lowering the interfacial energy of formation of the $\mathrm{NiSi}_{2}$ phase. In recent studies, comprehensive in situ TEM and SEM investigations of Ni silicate formation at various temperatures, growth directions, and nanowire diameters (with or without surface oxides), and by using $\mathrm{Ni}$ contacts pads as metal reservoirs, were demonstrated [29] (Figure 6(a)). The measurements showed retarded $\mathrm{NiSi}_{2}$ growth in the case of oxide-covered nanowires, but the reactions proceeded from the Ni reservoir (contact pad) and not from the opposite tip. Additionally, prolonged silicide growth in oxide-free nanowires resulted in formation of $\mathrm{Ni}$ rich phase closer to the $\mathrm{Ni}$ pads and massive volume expansion that caused wire breakages. In contrast, oxide-covered nanowires experienced no expansion and breakages during the silicide formation presumably due to the reduced Ni diffusion in Si caused by interfacial stress.

The formation of $\mathrm{Pt}$ and Co nanowire silicides has also been recently studied by in situ TEM of grown Si nanowires in contact with lithography defined pads [92, 93]. In general, similar trends during the growth of the silicides, dependent on the existence of a surface oxide and the nanowire growth directions, have been observed. For example, the growth of $\mathrm{PtSi}$ phase has been identified with the epitaxial relationships between $\mathrm{Si}$ and $\mathrm{PtSi}$ determined as $\mathrm{Si}(20-2) / / \mathrm{PtSi}(10-1)$ and $\mathrm{Si}[111] / / \mathrm{PtSi}[111]$, according to the diffraction patterns observed. In contrast, CoSi showed no epitaxial relationship with the Si nanowire and a much higher rate of formation. Similarly to the $\mathrm{NiSi}_{2}, \mathrm{CoSi}_{2}$ phase has also been identified at higher temperatures forming $\mathrm{Si}[110] / / \mathrm{CoSi}_{2}[110]$ and $\mathrm{Si}(111) / / \mathrm{CoSi}_{2}$ (111) epitaxial interfaces. Contact formation to $\mathrm{Ge}$ is in general much more challenging than in $\mathrm{Si}$ and has much more opened processing issue. This is partially due to the limited knowledge of the formation mechanism of metal germanides and in particular germanides grown as nanowires. To the best of our knowledge, there are only two nanowire germanides that are studied by in situ TEM, for example, $\mathrm{Ni}_{2} \mathrm{Ge}$ [94] and $\mathrm{Cu}_{3} \mathrm{Ge}$ [95]. The interest in $\mathrm{Cu}_{3} \mathrm{Ge}$ comes from the fact that it forms small energy barrier of just about $0.06 \mathrm{eV}$ to the valence band of Ge resulting in ideal ohmic contact for p-channel GeMOSFETs. In situ SEM studies at $310^{\circ} \mathrm{C}$ demonstrated that the germanide phase is propagating by forming a sharp interface with nonliner growth rate. Separate TEM measurements confirmed the resulting germanide phase and that there are $\mathrm{Ge}[-112] / / \mathrm{Cu}_{3} \mathrm{Ge}[-102]$ and $\mathrm{Ge}(1-11) / / \mathrm{Cu}_{3} \mathrm{Ge}(211)$ crystallographic relations between the germanide and the $\mathrm{Ge}$ nanowire. The TEM in situ crystallisation at $500^{\circ} \mathrm{C}$ of $\mathrm{Ni}$ germanides resulted in formation of $\mathrm{Ni}_{2} \mathrm{Ge}$ phase with a sharp interface with the $\mathrm{Ge}$ nanowire and $\mathrm{Ge}(111) / / \mathrm{Ni}_{2} \mathrm{Ge}$ 
(100) epitaxial relation. The estimated growth rates $(0.31 \mathrm{~nm} / \mathrm{s}$ and $1.05 \mathrm{~nm} / \mathrm{s}$ ) suggested almost linear behaviour at 400 and $500^{\circ} \mathrm{C}$ for the two different temperatures, respectively. It should be mentioned that in both studies, the surface oxides were intentionally removed before metal evaporation.

The reactive epitaxial silicide or germanide growth is unlikely to proceed as the molecular beam epitaxy and certainly requires full atomistic understanding and further study at high resolution. Until now, lattice resolution imaging in real time has only been observed on several occasions of the propagating Ni silicide crystals $[29,92]$. The growth proceeds in a plane-by-plane lattice formation, and in some cases, there is a formation of stepped interfaces (see Figure 6(b)) [29]. It is interesting to note that the layer-by-layer lattice growth is not continuous, but it is accompanied by intervals between the steps which vary in time.

In brief, it can be concluded that the overall growth rate of nanowire silicide and germanide is ruled out by the competition of metal diffusion and interface reactions along the nanowire; the rate limiting factor is still unclear. It depends on the surface oxide and associated stress as well as the phase formed at the interface, minimising the formation energy and the lattice mismatch. It is generally seen that metal rich phases are seen closer to the metal reservoirs. It is surprising, although not studied in detail, that the overall growth rate does not depend on the nanowire diameters, as it might be expected that in very small diameter nanowires, the surface defects might influence the metal diffusion considerably as well as the formation of the propagating silicide or germanide interface. Another fully unexplored field of in situ TEM studies is in the determination of the silicide or germanide formation as a function of the level and type of doping in the nanowires. Notably, all the discussed studies deal with unintentionally doped structures, although for n-type Ge nanowires, for example, there are no solutions to reliable contact formation yet.

3.2.2. Nanowire Surface Regrowth, Implantation Damage, and Recrystallisation Observed by In Situ TEM. Besides the studies of the transformations during formation of contacts to semiconductor nanowires, the understanding of nanowire surface properties has an even greater role in the nanowire device performance [87]. Small diameter semiconductor nanowires have large surface area-to-volume ratio, and carrier scattering and recombination at surface defects (traps) may significantly compromise the electronic and optoelectronic properties of nanowire devices. Specifically, the performance of electronic devices such as FETs based on Ge nanowires is largely dominated by this phenomenon. Therefore, heteroepitaxial growth of an Si or SiGe alloy shell around a Ge nanowire core has previously been explored to achieve surface passivation and carrier confinement, in part by promoting the formation of a stable outer $\mathrm{Si} / \mathrm{SiO}_{2}$ interface and by creating a core-shell band offset [96]. Postgrowth thermal processes can affect surface morphology, coherency strain, and chemical composition of core-shell heterostructures. The thermal evolution of these parameters was recently investigated by in situ TEM heating experiments [97]. It has been demonstrated that the strain relaxation mechanisms during postgrowth annealing include $\mathrm{Si}-\mathrm{Ge}$ interdiffusion, stress-driven surface roughening, and associated dislocation formation. At $700^{\circ} \mathrm{C}$, the nanowire surface increased in roughness at the initial stages of the surface regrowth, which was followed by abrupt reduction of the surface roughness until the nanowire surface became comparable with its initial state. These transformations were attributed to the intermixing of the initial SiGe shell with the underlying core as measured by line-scan EDS analysis across the nanowire. Interestingly, smaller diameter nanowires showed similar dynamic behaviour. In contrast, transformations observed at reduced temperature of $550^{\circ} \mathrm{C}$ showed almost no roughening and elemental intermixing at the surface. The associated defect formation was determined after the dynamic measurements and showed that the majority of the defects are $\langle 110\rangle$ perfect dislocations and intrinsic stacking faults bounded by $\langle 112\rangle$ Shockley partial dislocations. Unfortunately, the dynamics of the core/shell intermixing and associated stress evolution was difficult to measure at high resolution. Most importantly, it has been demonstrated that in situ TEM heating experiments can provide vital information regarding nanowire surface regrowth and reorganisation that can have detrimental effects on nanowire-based device properties.

Addressing the needs of future device failure analysis and metrology, the aim will be to develop capabilities for in-situ TEM analyses of nanowires interfaced as part of device prototypes. For example, a CMOS-compatible device architecture such as multigate nanowire FET, manufactured using Silicon-on-insulator (SOI) platform, will require three electrical terminals that are interfaced within the TEM specimen arm. In this case, the evolution of the developed contacts or nanowire surfaces can be observed while the electrical responses are registered. Such studies can answer many questions regarding the very debatable role of the contact formation and the surface states related to the formation of reliable and high performance nanowire devices. In order to study the result of these structural changes over the properties of the nanowire devices, an in situ TEM set-up that allows for simultaneous or sequential measurements of the structural and electrical changes "on-site" is required. As mentioned in the previous section of this paper, our approach is to devise an electrical TEM holder and sample preparation procedure capable of measuring devices using at least three electrical terminals. In the best case scenario, such devices would be developed by processes that allow manufacturing compatible with current semiconductor technology and that can be electrically tested by conventional electrical probe stations for comparative purposes and benchmarking.

Another important aspect of nanowire-based electronic devices such as FETs is the formation of nanostructured junctions. Nanowires can be doped in situ during growth, including formation of radial or axial junction heterostructures [98]. On the other hand, ion implantation is the industry preferred option for doping and junction formation for semiconductor devices. Ion implantation of nanowires and subsequent thermal treatment to cure defects and activate dopants have been studied only postfactum for Si and Ge nanowires $[99,100]$ as well as lithography defined Si and Ge thin-body structures $[101,102]$. By implanting Si nanowires in 
several steps with different implantation parameters, uniform doping profiles as well as $\mathrm{p}-\mathrm{n}$ junctions were achieved. The successful activation of the dopants is demonstrated by electron beam-induced current (EBIC) imaging and two point current-voltage measurements by using in situ contacting within the SEM [99]. Similarly, it has been demonstrated that with Ga-ion implantation, Ge nanowires show intriguing electrical performance as p-type nanowire-based FETs without the need for thermal activation in contrast to $\mathrm{Si}$ nanowires [100]. Unfortunately, detailed analysis of implantinduced damage and its evolution by thermal annealing has not been presented. These effects are indeed important when dealing with thin-body structures such as nanowires or fins as predicated by density, functional theory, and dynamic Monte-Carlo simulations and observed by ex situ lattice resolution TEM imaging [103]. At the sidewall surfaces, the crystal lattice is irregular, and consequently the formation of distorted bonds, respectively defects during annealing, is promoted. As a result, it is expected that stacking fault defects originate at the surfaces due to disturbance of templated atom incorporation and propagate into the bulk. Notably, in situ ion implantation TEM capabilities have not yet been used to study nanowire materials. Only very recently, we have initiated in situ TEM experiments to observe implantationinduced damage in Ge nanowires and fins and their thermal evolution by in situ vacuum annealing. Briefly, our investigations are based on performing repetitive (semi-in situ) Ga-ion implantations and structural imaging of the same Ge nanowire by using dedicated platform for transferring between a Dual Beam SEM/FIB instrument and a TEM. This is followed by examining the defect evolution using in situ TEM heating stage [104]. We have demonstrated that there is a threshold in the Ga-ion dose for full amorphisation of the nanowires which is dependent on the nanowire diameter and the orientation of the nanowires towards incident beams. The rate of lattice damage is dependent on the ion fluence used, and its impact is reduced with number of repetitive implantations. Full thermal curing of partially amorphous $\mathrm{Ge}$ nanowires is possible at temperatures as low as $300^{\circ} \mathrm{C}$ (including full recrystallisation of already existing defects). Fully amorphous nanowires recrystallise as polycrystalline.

\subsubsection{In Situ TEM Annealing Studies of Nanowire-Based} Phase Change Materials. Phase change memory devices based on nanowires received increased attention due to their intrinsically miniaturised dimensions and the opportunity to manufacture high density arrays. Nanowires made of phase change materials are attractive for such applications as it is predicted that by decreasing the volume of the active phase change material, the overall power consumption of the device will be reduced [105]. In order to understand the transitions and material failure of GeTe nanowires as building blocks for phase change memory devices, the nanowires were coated with an oxide shell and vacuum annealed within the TEM [106]. The results indicated considerable suppression of the melting point (below $500^{\circ} \mathrm{C}$ ) localised at the tips of the nanowires near the Au catalyst particles. The transformations occurred at temperatures considerably lower than the melting temperature of GeTe and were attributed not only to the nanowire dimensions but also to the formation of GeTe-Au eutectic. In comparison, the pure GeTe part of the nanowires transforms by sublimation without forming a liquid eutectic rather than by forming facets at the nanowire ends.

A different study examined phase changes in $\mathrm{Ag}_{2} \mathrm{Te}$ nanowires, a material with intriguing thermoelectric and magnetoresistive properties [107]. Monoclinic $\mathrm{Ag}_{2} \mathrm{Te}$ nanowires transform to face-centred and finally bodycentred cubic phases as the temperature is increased, accompanied by changes in resistivity and lattice volume. Using in situ TEM annealing of grown $\mathrm{Ag}_{2} \mathrm{Te}$ nanowires, the thermal evolution was followed by small area electron diffraction at the nanowire tips. The obtained dynamic diffraction at the temperature interval of 120 to 180 (at $10^{\circ} \mathrm{C}$ increments) revealed smooth transitions from monoclinic to face-centred cubic phase. Upon cooling, the structure fully recrystallised in its original monoclinic phase but at slightly higher temperature of $120^{\circ} \mathrm{C}$, demonstrating a temperature hysteresis in the phase transition. Additionally, the changes in the $\mathrm{Ag}_{2} \mathrm{Te}$ phases were studied under compressive stress by encapsulating $\mathrm{Ag}_{2} \mathrm{Te}$ nanocrystals and thermally expanding them in Pt cladding layers. Surprisingly, at $130^{\circ} \mathrm{C}$, the monoclinic phase started to transit to a high-temperature body-centred cubic preferentially along the boundaries of the nanowire, instead of the expected lower temperature face-centred cubic phase. In the bulk of the nanowire, the transformation to face-centred cubic phase dominated. Finite element analysis was used to model the compressive stress distribution. It is suggested that the reason for this unusual transformation is the existence of high compressive stress at the nanowire boundaries. These results exemplify the importance of using in situ TEM methodology to observe abnormal phenomena associated with the large surface to volume ratio in nanoscale materials. The explanation of this unusual behaviour was ascribed to the nanoscale dimensions combined with the small loading area inducing significant pressure effects; the result is stabilisation of otherwise energetically improbable phase transitions.

Until now, we have discussed how growth, phase transitions, and recrystallisation of nanowires and nanotubes can be observed by using in situ E-TEM and in situ TEM annealing. These processes are fundamentally important because they reveal in great detail the discreet phenomenological features of the crystal growth, phase transitions, defects, and surface transformations at the nanoscale. Although essential, these studies can only be used as directors for accessing the performance of the nanowire structures as functional devices. Direct measurement of the function of nanowires is possible by assembling and interfacing a nanowire-based device within the electron microscope. In this case, multimode analysis required is capable of detecting multiple signals, such as electrical, electromechanical, light, or other within the TEM.

\subsection{Electrical Interfacing and Manipulation of Nanowires} within the TEM. The first examples of using electrical probes to assemble and operate nanowire devices within a TEM have been demonstrated more than 10 years ago. Since 
then, various nanowire and nanotube materials have been subjected to analysis by this approach with the main focus being on (i) electrical characterisation accompanied by observation of the devices at extreme spatial resolution and (ii) observing transformations and action of a nanowire by applying electrical stimuli. In the first case, two-point electrical measurements (devices with more than two terminals have not yet been realised) are performed, while the nanowire structure and contacts are imaged simultaneously. This mode of operation is equivalent to nanowire electrical testing with added capability of direct observation of nanowire structure and points of electrical contacts. The second approach is more complex and uses the in situ TEM electrical contacting capabilities to manipulate the nanowires and measure the corresponding nanowire transformations. Technically, these studies can be accomplished by using dedicated stages for micro- or nanocontacting designed for SEMs or TEMs as described previously.

\subsubsection{Electrical Interfacing of Semiconductor Nanowires and} Nanotubes. When measuring the electrical performance of the nanowires or nanotubes by two-terminal electrical contacts within the TEM, the major question is about the quality of the contacts. The contact between the piezodriven metal terminal and the nanowire (nanotube) and its evolution during biasing can be directly visualised by using TEM imaging (see Figure 7(a)). Unfortunately, the topology of the opposite contact is usually unknown as the nanowires are dispersed and attached by conductive glue to this terminal. Thus, it is often seen that the electrical data reflects problems with nanowire contact resistances manifested in nonohmic $I-V$ curves. Special care needs to be taken for removing surface contamination, surface oxides, or inhomogeneities that act as barriers for charge carriers. This can be achieved by various means such as (i) chemical or physical etching of the metal contacts and/or nanowire materials immediately before contacting [32], (ii) applying high floating bias on the micron-sized terminal that induces localised heating [108], (iii) fine mechanical rubbing using nanoprobe terminal [30], or (iv) deposition of additional conductive material by site-specific electron beam-induced deposition [109]. Improvement of (ohmic) electrical contacts to CNTs or semiconductor nanowires using such techniques has been demonstrated (Figure 7(a), inset). In the future, introducing additional terminals during the measurement and better control over the electrical contact formation may advance the practicality of this technique.

The structural transformations, both at the nanowire contacts or within the nanowire/nanotube body during electrical biasing at high potentials (high electrical power), can result in localised thermal heating (Joel heating) and mass transport due to thermoelectric migration. The effects associated to these phenomena are annealing induced sintering, recrystallisation, and formation of new phases at the contacts or within the nanowire/nanotube structure. Mass transport due to thermoelectric migration involves motion of atoms from cathode to anode, imparted by the momentum of electron wind due to high current density, with the associated creation of vacancies and subsequent amorphisation. This process will not take place to a large extent if the anode can supply vacancies and the cathode can accept them continuously. Nevertheless, in situ TEM electrical studies of lithographically defined polycrystalline $\mathrm{Al}$ interconnects reveal that even relatively low current densities can lead to amorphisation due to thermoelectric migration [110]. Future studies are needed to further understanding of this phenomenon and to investigate in detail semiconductor devices featuring ultimately miniaturise nanowire interconnects and associated contacts. In this case, mass-transport effects due to electromigration can be extremely destructive, and the possibility to observe them in situ will result in optimised device performance and address possible reliability issues.

Resistive heating of a nanowire or a nanotube can lead to structural transformations equivalent to what can be seen when the structures are annealed using in situ TEM heating stage. Indeed, these can be visualised by imaging the point of contact of the nanoprobe with the nanowire or the nanotube after applying high potential. An important question when performing in situ thermal experiments with resistively heated specimens is regarding the exact temperature on the sample surface. Recently, a valuable method to measure the temperature on the sample surface has been demonstrated [111]. It uses microfabricated metal lines on electron transparent silicon nitride membranes that can carry high electrical currents. The sample surface was decorated by Au metal nanoparticles that changed their morphology upon heating and were used as sample in situ thermometers. Melted nanoparticles form unique temperature profile around the metal line, thus visualising the temperature gradient on the surface of the silicon nitride membrane. The obtained results agree very well with the finite element modelling of temperature distribution around a metal line.

By using the electrical nanoprobe to resistively heat individual structures, it has been demonstrated that CNTs structure can be rectified by removing amorphous carbon material or nanotube kinks [112]. Formation of onion-type carbon, metal carbides, and diffusion of catalytic metal nanoparticles within the tube interior as well as deformation of metal nanocontact have also been observed by this technique $[54,113]$. Similarly, Si, Ge, and compound semiconductor nanowires have been contacted and resistively heated. To the best of our knowledge, formation of metal silicides or germanides has not been observed by this method. We, and others, have observed formation of segmented Ge nanowires within a Ge oxide shell as a result of excessive electrical power [32]. Notably, this has been observed only with nanowires having Ge oxide and poor electrical conductivity. The mechanism of this transformation has not been fully elucidated, but it is suggested that it is associated with masstransport induced breakages due to electrothermal migration and not Ge melting due to resistive heating.

A different approach for contacting and simultaneous electrical testing was presented by Kallesøe and coworkers [31]; the electrical contacting of Si nanowires was controlled by influencing the epitaxial growth of Si nanowires formed on conductive Si cantilever loops by Joel heating (Figure 7(b)). Briefly, the metal catalysts were deposited by Au evaporation 


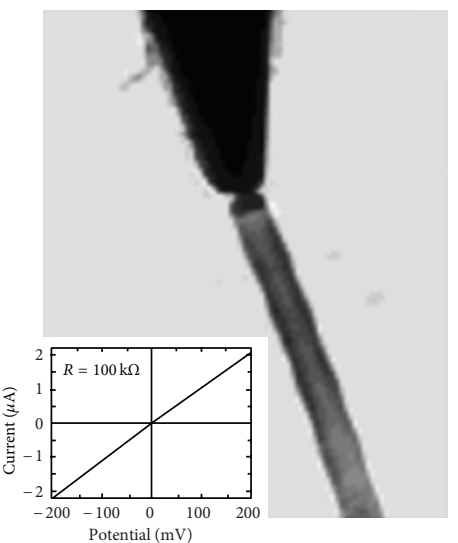

(a)
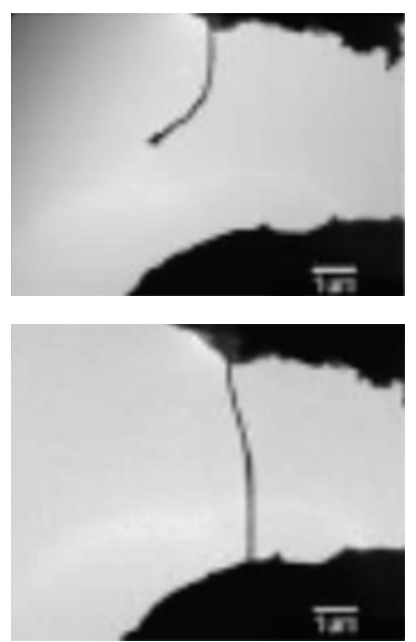

$\overline{1}$
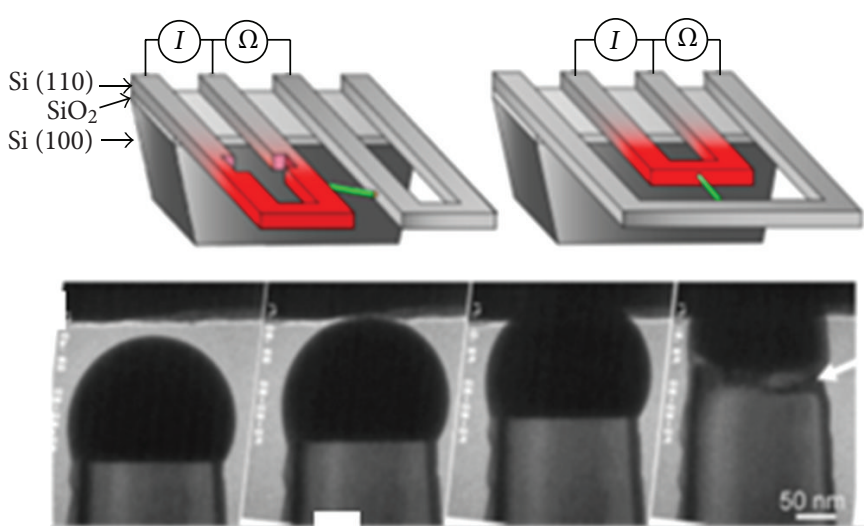

(b)
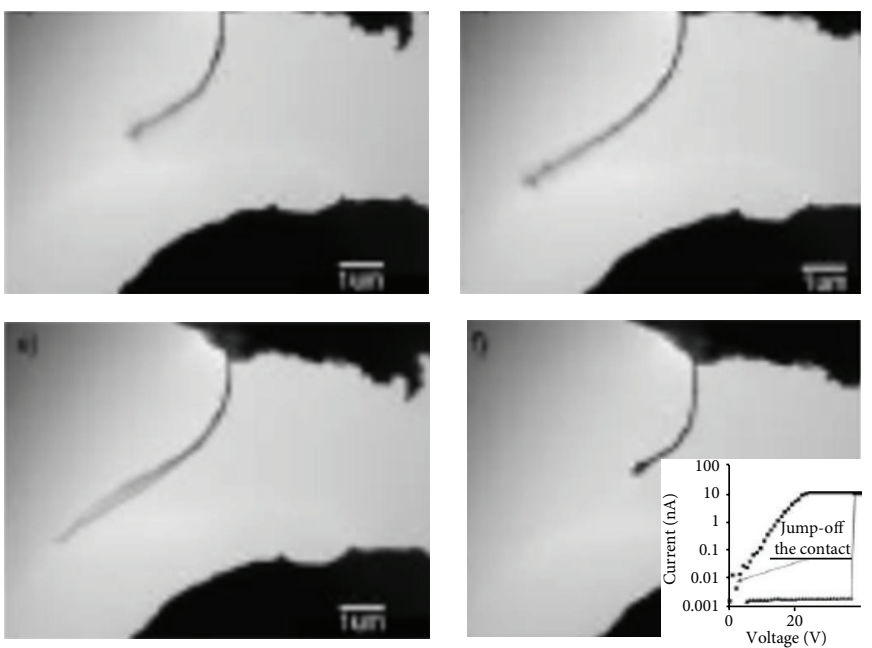

(c)
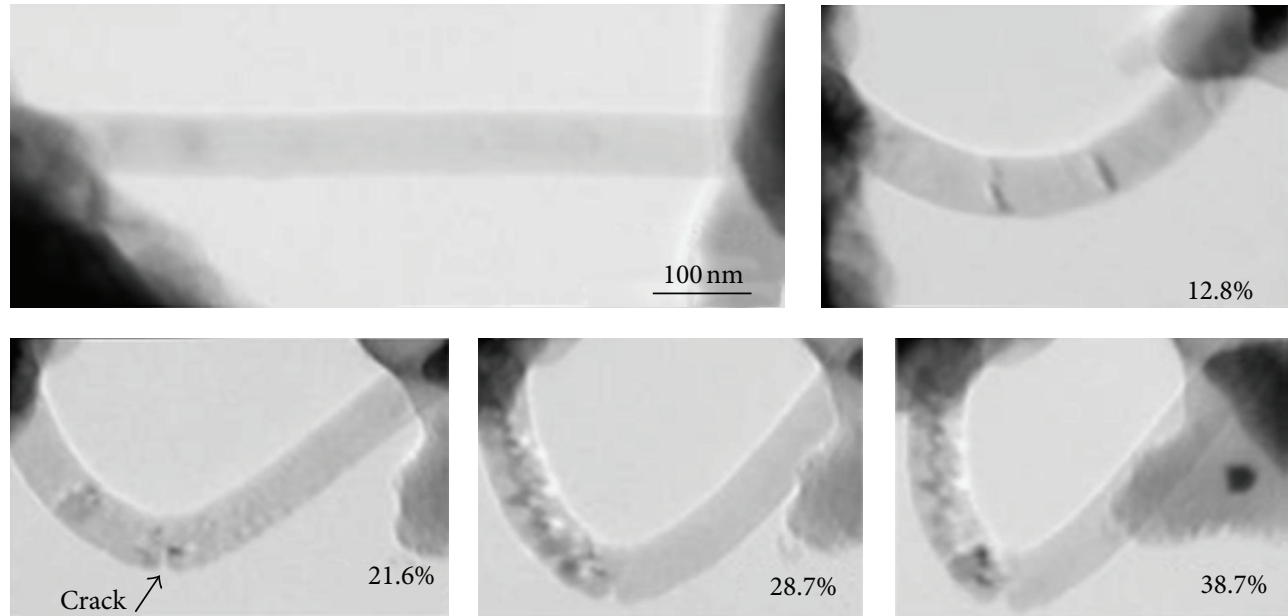

(d)

FIGURE 7: (a) TEM image of tow-terminal nanowire device using piezo-driven nanocontacting, inset corresponding $I$ - $V$ curve, adapted from [30]. (b) Schematics of the microfabricated resistively heated loops used to grow and contact Si nanowires and corresponding sequence of TEM images showing a nanowire approaching and contacting, adapted from [31]. (c) Sequence of TEM images demonstrating the function of Ge nanowire-based NEMs device, inset: corresponding I-V curve showing sharp drop in current at jump-off the contact, adapted from [32]. (d) Sequence of TEM images of an Si nanowire subjected to bending stress demonstrating the formation of large number of defects, adapted from [33]. 
in UHV chamber after removing the native oxide, and the growth temperature is maintained by resistive heating of microfabricated cantilever loops. Arrhenius dependence of the growth rate to the temperature was found, allowing for calibration of the temperature distribution along the heating loops by measuring the wire lengths. The growing nanowires were brought into contact to an additional nearby contacting loop, and the obtained devices were tested electrically. Remarkably, when the contacting loop is kept cold by having no current flowing through the loop, the approaching metal tip of the growing nanowire changed its shape and solidified, forming $\mathrm{Si}-\mathrm{Au}-\mathrm{Si}$ contacts. The change of the shape of the metal tip is explained by the surface tension, which reformed the liquid metal droplet when being in contact with the surface of the contacting loop. Such contacts showed nonohmic highly resistive $I-V$ curves. When the contacting loop was resistively heated, the Au nanoparticle on the tip of the growing NW migrated away, leaving Si-Si in contact with better electrical performance. Certainly, such in situ TEM studies are difficult to execute and control; moreover, gating of the devices is not possible and requires at least three electrical terminals.

\subsubsection{Electrical Interfacing and Transformations of Nanowires} as Electrodes in Li-Ion Batteries. Another type of nanowire device that has been recently assembled using the capabilities of the in situ electrical probing TEM stage is a nanobattery. The study of phase transitions during the operation of Liion batteries has gained importance as cells with higher energy and power densities, better cyclability, and durability are now in demand [114]. A major problem with the anode materials (industry standard is graphite) is reduced structural integrity upon cycling; consequently, it has been suggested that the graphite anode can be replaced by nanowire materials [115]. With the development of the electrical contacting capabilities for TEM, it has become possible to observe, in real time, structural transformations associated with Li-ion charge/discharge reactions within single nanowire electrochemical cells called nanobatteries. Liu and Huang were the first to demonstrate the assembly and operation of such a cell, hence the major advances in this field have been recently reviewed by the authors[11]. Briefly, all solid-state electrochemical cells can be assembled by attaching nanowires on a metal contact tip that is brought into contact with metal Li covered by solid-state electrolyte. The nanowire and nanotube materials that have been studied using this approach include $\mathrm{Si}$ and Ge NWs [116, 117], CNTs and graphene [11], $\mathrm{ZnO}, \mathrm{CuO}$ [118], $\mathrm{SnO}_{2}$ [11], and pure $\mathrm{Al}$ nanowires [119]. The transformation mechanism during lithiation of the anode material composed of a nanowire normally starts at the contact of the nanowire tip with the electrolyte, and it propagates along the nanowire axis. The exact morphological changes of the nanowire anode are largely dependent on the nanowire composition, but in all cases, there are a large volume expansion and associated electrochemically driven solid-sate amorphisation. For example, $\mathrm{SnO}_{2}$ nanowires are amorphised during lithiation by initially forming a large number of mobile dislocations also known as "Medusa zone," while $\mathrm{Si}$ and Ge nanowires are transformed by formation of amorphous $\mathrm{Li}_{x} \mathrm{Si}$ or $\mathrm{Li}_{x} \mathrm{Ge}$ shells on the surface that increase in thickness with the lithiation time, which in the end transform into crystalline $\mathrm{Li}_{15} \mathrm{Si}_{4}$ or single crystalline $\mathrm{Li}_{15} \mathrm{Ge}_{4}$ phase. The $\mathrm{Al}$ nanowires show volume expansion during lithiation which is, in contrast to $\mathrm{SnO}_{2}$ and $\mathrm{Si}$ nanowire anodes, associated with formation of a large number of voids along the whole nanowire length. Similarly, a multiwalled carbon nanotube was subjected to lithiation resulting in circumferential expansion and disorder in tube wall. The lithiation-induced embrittlement of the tube that has not been seen before in stark contrast to the extreme flexibility of the pristine nanotubes. The delithiation process in nanowires and nanotubes does not cause reversible transformations in their structure. In contrast to phase change materials, the transformations may result in formation of voids or an interwoven porous network as in the case of $\mathrm{Al}$ and Ge nanowires. Intriguingly, the porous nanowires exhibited fast lithiation/delithiation rates and excellent mechanical robustness, attributed to the high rate of lithium diffusion and the porous network structure for facile stress relaxation, respectively.

3.3.3. Performance of Nanowire-Based Mechanical and Electromechanical Systems Observed within the TEM. In an attempt to follow Ge nanowire performance as nanoelectromechanical systems (NEMS), we have explored the possibility of assembling two-terminal devices based on Ge nanowires attached to a piezo-driven contact within the TEM (Figure 7(c)) [32]. The function of the device is based on delicately balancing electrostatic, elastic, and adhesion forces between the nanowires and the contacts, which can be controlled by the applied voltage. The advantage of using in situ TEM technique is that the operation of the NEM device including the switching between the ON-OFF states can be visualised directly during the experiments. Additionally, the position of the contacts and the length of the nanowire, from the clamping point to the contact with the opposite electrode, can be adjusted within the system without the need to prepare new specimens. This level of flexibility and fast control cannot be achieved when NEM devices are prepared by electron beam lithography and other techniques. We have demonstrated that the switching processes in our NEM devices are strongly dependent on the nanowire diameters, existence of surface oxide, and most importantly well-developed single crystalline structure of the Ge nanowires. In comparison to NEM devices based on multiwalled CNTs measured under similar conditions, Ge nanowires present a much more robust and reliable NEM system.

The mechanical properties of nanowires or nanotubes such as Young's and bending modulus can be estimated by applying alternating potential on one electrical terminal with structures attached to it $[7,120,121]$. In these cases, the nanowires or nanotubes act as cantilevers. When no potential is applied, the nanowire or CNT cantilever will start to oscillate due to thermal effects. After applying alternating potential to the CNT, the fundamental mode of vibration as well as its first harmonic can be excited and 
visualised by the TEM; the frequencies and the shapes of the vibration modes demonstrated that the CNT acts as a uniformly cantilevered beam [120]. According to the classical elasticity theory, the resonant frequencies are dependent on the CNTs diameter and proportional to the bending modulus, hence the bending modulus of CNTs can be determined by observing the resonant frequencies by in situ TEM. Another approach is to use in situ TEM straining stages $[19,33,59-64]$. Single crystalline $\mathrm{Cu}$ domains deposited on a microfabricated MEMs stage were subjected to uniaxial strain while observing the structure at high resolution [122]. It was demonstrated that large elasticity, reaching ideal elastic limit, can be sustained by elongation of the crystal lattice along the [001] direction. Similarly, using AFM set-up within the TEM, grown Si nanowires were elongated along their growth direction and showed rapture without noticeable formation of defects [33]. The raptured surface appeared perfectly cleaved along the [111] set of planes. Moreover, it has been demonstrated that the rapture strength increases with decreasing nanowire diameters. In stark contrast, when bending stress was applied, the Si nanowires were deformed by forming large number of dislocations, followed by partial amorphisation and final cracking (Figure $7(d)$ ). Remarkably, the nanowires sustained large amounts of bending strain (more than 20\%-25\%) before complete failure.

\section{Conclusions and Outlook}

In conclusion, the present paper highlighted recent advances in the in situ TEM instrumentation and operation conditions which made the discovery of significant scientific findings possible regarding the dynamics of the morphology, structure, and composition of one-dimensional materials. By presenting detailed discussion of several unique cases, it is demonstrated how obtained dynamic data can be used to reevaluate general formulation used to describe growth and crystallisation thermodynamics and kinetics by accounting for phenomenological differences, for example, stabilisation of otherwise energetically improbable phase transitions due to the nanoscale dimensions and/or far from equilibrium growth. Furthermore, studies where direct measure of the function of one-dimensional nanostructures within the TEM are highlighted, pinpointing the importance of the capabilities for in situ assembling and interfacing a nanowire-based devices.

Technologically, the in situ TEM technique will play increasingly important role in discovering novel transformation phenomena associated with nanoscale dimensions in materials for which the growth and processing technology is less mature. It is well documented that while Si has dominated the semiconductor industry for many decades, alternative materials (Ge, III-Vs, CNTs, and graphene) with nanoscale dimensions are rapidly emerging as realistic competitors and potential novel "building blocks" for revolutionary semiconductor technologies as outlined in the recent ITRS roadmap. Similarly, such materials will play even great role in addressing challenges in energy and environmental applications. The difficulty is that such materials require immerging or in some cases novel growth and processing technologies such as, for example, laser thermal annealing (LTA) versus conventional rapid thermal annealing (RTA), alternative doping or deposition technologies, or devising routes for defects-free growth of epitaxial layers and others. For such processes, emerging materials and device architectures detailed understanding of the transformations under external stimuli at ultimate resolution is required and can be supplied by in situ TEM.

On the other hand, it is clear that until now only a small fraction of the developed growth and processing techniques have found their route to detailed mechanistic examination by the in situ TEM technique. Among them, solvothermal growth conditions, although very popular, seem probably the most unrealistic for in situ TEM investigation. These will require novel design of the liquid cell by introducing, for example, localised heating elements, mixing valves within the microfluidic channels, and electron transparent windows that can sustain high vapour pressure. If developed, they will benefit the understanding of the growth mechanism for semiconductor and oxide structures, such as, for example, $\mathrm{TiO}_{2}$ nanowires and nanotubes, nanosized zeolites, and metal and oxide nanoparticles, that are normally obtained by solvothermal growth.

Increasing the complexity of the in situ TEM technique, multimodal operation capable of applying and detecting multiple signals in situ (besides those triggered by the electron beam itself, taking part in the TEM analysis) will gain importance. In situ TEM operations based on the multimode approach that has not yet been demonstrated and that if developed can strongly benefit the analysis as well as validate the function of electronic and optoelectronic devices include

(i) observing transformations within a fully interfaced device while applying mechanical or electrical stress and recording in parallel its electrical or optical response,

(ii) oppositely, following transformations in a device that is electrically pumped or mechanically strained and being able to detect in situ its light emission properties,

(iii) recording spatially resolved cathodoluminescence (CL) signals from fully integrated device while applying a mechanical strain.

The significance of these approaches can only be seen if the associated in situ TEM instrumentation is developed with improved flexibility and adaptability to different types of samples. From future metrology and device quality control perspective, the demand will be in developing capabilities for in situ TEM analyses of structures interfaced as a part of prototype devices manufactured by industry compatible technologies and not simple random nanowires as usually achieved. 


\section{References}

[1] K. W. Urban, "Is science prepared for atomic-resolution electron microscopy?" Nature Materials, vol. 8, no. 4, pp. 260-262, 2009.

[2] O. L. Krivanek, M. F. Chisholm, V. Nicolosi et al., "Atom-byatom structural and chemical analysis by annular dark-field electron microscopy," Nature, vol. 464, no. 7288, pp. 571-574, 2010.

[3] G. Dehm, J. M. Howe, and J. Zweck, Eds., In situ Electron Microscopy: Applications in Physics, Chemistry and Materials Science, Wiley-VCH GmbH \& Co. KGaA, Weinheim, Germany, 2012.

[4] In situ Electron Microscopy at High Resolution, World Scientific Publishing, 2008.

[5] P. L. Gai, R. Sharma, and F. M. Ross, "Environmental (S)TEM studies of gas-liquid-solid interactions under reaction conditions," MRS Bulletin, vol. 33, pp. 107-114, 2008.

[6] R. Sharma, "Kinetic measurements from in situ TEM observations," Microscopy Research and Technique, vol. 72, no. 3, pp. 144-152, 2009.

[7] X. Han and Z. Zhang, "Experimental nanomechanics of onedimensional nanomaterials by in situ microscopy," NANO: Brief Reports and Reviews, vol. 2, pp. 249-271, 2007.

[8] Q. Chen and L.-M. Peng, "Fabrication and electric measurements of nanostructures inside transmission electron microscope," Ultramicroscopy, vol. 111, pp. 948-954, 2001.

[9] D. Golberg, P. M. F. J. Costa, M.-S. Wang et al., "Nanomaterial engineering and property studies in a transmission electron microscope," Advanced Materials, vol. 24, pp. 177-194, 2012.

[10] N. de Jonge and F. M. Ross, "Electron microscopy of specimens in liquid," Nature Nanotechnology, vol. 6, pp. 695-704, 2011.

[11] X. H. Liu and J. Y. Huang, "In situ TEM electrochemistry of anode materials in lithium ion batteries," Energy and Environmental Science, vol. 4, pp. 3844-3860, 2011.

[12] A. H. Zewail, “4D electron microscopy,” Science, vol. 328, p. 187, 2010.

[13] E. P. Butler and K. F. Hale, "Dynamic experiments in the electron microscope," in Practical Methods in Electron Microscopy, A. M. Glauert, Ed., vol. 9, Elsevier, Amsterdam, The Netherlands, 1981.

[14] E. D. Boyes and P. L. Gai, "Environmental high resolution electron microscopy and applications to chemical science," Ultramicroscopy, vol. 67, no. 1-4, pp. 219-232, 1997.

[15] http://hitachi-hta.com/products/electron-microscopes-andfocused-ion-beam/transmission-electron-microscopes/h9500-300kv-te.

[16] http://www.cse.salford.ac.uk/sumc/2000fx.php.

[17] http://www.gatan.com/products/specimen_holders/products/ 628-Single-Tilt-Heating-Holder.php.

[18] http://www.denssolutions.com/en/our-products/sample-heating-systems.

[19] Y. Zhu and H. D. Espinosa, "An electromechanical material testing system for in situ electron microscopy and applications," Proceedings of the National Academy of Sciences of the United States of America, vol. 102, pp. 14503-14508, 2005.

[20] http://www.nanofactory.com/news.asp?id=59\&type=news.

[21] J. M. Grogan, L. Rotkina, and H. H. Bau, "In situ liquidcell electron microscopy of colloid aggregation and growth dynamics," Physical Review E, vol. 83, no. 6, Article ID 061405, 2011.
[22] B. Westenfelder, J. C. Meyer, J. Biskupek et al., "Graphene-based sample supports for in situ high-resolution TEM electrical investigations," Journal of Physics D, vol. 44, no. 5, Article ID 055502, 2011.

[23] J. M. Yuk, J. Park, P. Ercius et al., "High-resolution EM of colloidal nanocrystal growth using graphene liquid cells," Science, vol. 336, pp. 61-64, 2012.

[24] S. Hofmann, R. Sharma, C. Ducati et al., "in situ observations of catalyst dynamics during surface-bound carbon nanotube nucleation," Nano Letters, vol. 7, no. 3, pp. 602-608, 2007.

[25] S. Hofmann, R. Sharma, C. T. Wirth et al., "Ledge-flowcontrolled catalyst interface dynamics during Si nanowire growth," Nature Materials, vol. 7, no. 5, pp. 372-375, 2008.

[26] S. Kodambaka, J. Tersoff, M. C. Reuter, and F. M. Ross, "Germanium nanowire growth below the eutectic temperature," Science, vol. 316, no. 5825, pp. 729-732, 2007.

[27] E. A. Sutter and P. W. Sutter, "Size-dependent phase diagram of nanoscale alloy drops used in vapor-liquid-solid growth of semiconductor nanowires," ACS Nano, vol. 4, no. 8, pp. 49434947, 2010.

[28] Y. C. Lin, Y. Chen, D. Xu, and Y. Huang, "Growth of nickel silicides in $\mathrm{Si}$ and $\mathrm{Si} / \mathrm{SiO}_{x}$ core/shell nanowires," Nano Letters, vol. 10, no. 11, pp. 4721-4726, 2010.

[29] K. Ogata, E. Sutter, X. Zhu, and S. Hofmann, "Ni-silicide growth kinetics in $\mathrm{Si}$ and $\mathrm{Si} / \mathrm{SiO}_{2}$ core/shell nanowires," Nanotechnology, vol. 22, no. 36, Article ID 365305, 2011.

[30] M. W. Larsson, L. Reine Wallenberg, A. I. Persson, and L. Samuelson, "Probing of individual semiconductor nanowhiskers by TEM-STM," Microscopy and Microanalysis, vol. 10, no. 1, pp. 41-46, 2004.

[31] C. Kallesøe, C. Y. Wen, T. J. Booth et al., "in situ creation and electrical characterization of nanowire devices," Nano Letters, vol. 12, pp. 2965-2970, 2012.

[32] J. Andzane, N. Petkov, A. I. Livshits, J. J. Boland, J. D. Holmes, and D. Erts, "Two-terminal nanoelectromechanical devices based on germanium nanowires," Nano Letters, vol. 9, no. 5, pp. 1824-1829, 2009.

[33] D.-M. Tang, C.-L. Ren, M.-S. Wang et al., "Mechanical properties of Si nanowires as revealed by in situ transmission electron microscopy and molecular dynamics simulations," Nano Letters, vol. 12, pp. 1898-1904, 2012.

[34] C. M. Lieber and Z. L. Wang, "Functional nanowires," MRS Bulletin, vol. 32, pp. 99-108, 2007.

[35] A. P. Graham, G. S. Duesberg, W. Hoenlein et al., "How do carbon nanotubes fit into the semiconductor roadmap?" Applied Physics A, vol. 80, no. 6, pp. 1141-1151, 2005.

[36] R. G. Hobbs, N. Petkov, and J. D. Holmes, "Semiconductor nanowire fabrication by bottom-up and top-down paradigms," Chemistry of Materials, vol. 24, no. 11, pp. 1974-1991, 2012.

[37] O. Haydena, R. Agarwalb, and W. Lu, "Semiconductor nanowire devices," Nanotoday, vol. 3, pp. 12-22, 2008.

[38] F. Patolsky, G. Zheng, and C. M. Lieber, "Nanowire-based biosensors," Analytical Chemistry, vol. 78, no. 13, pp. 4261-4269, 2006.

[39] D. C. Bell, Y. Wu, C. J. Barrelet et al., "Imaging and analysis of nanowires," Microscopy Research and Technique, vol. 64, no. 5-6, pp. 373-389, 2004.

[40] R. Zan, Q. M. Ramasse, U. Bagert, and K. S. Novoselov, "Graphene reknits its holes," Nano Letters, vol. 12, pp. 39363640, 2012. 
[41] T. LaGrange, G. H. Campbell, B. W. Reed et al., "Nanosecond time-resolved investigations using the in situ of dynamic transmission electron microscope (DTEM)," Ultramicroscopy, vol. 108, no. 11, pp. 1441-1449, 2008.

[42] A. H. Zewail and J. M. Thomas, 4D Electron Microscopy: Imaging in Space and Time, World Scientific Publishing, 2010.

[43] I. M. Abrams and J. W. McBrain, "A closed cell for electron microscopy," Journal of Applied Physics, vol. 15, pp. 607-609, 1944.

[44] P. L. Gai and K. Kourtakis, "Solid-state defect mechanism in vanadyl pyrophosphate catalysts: implications for selective oxidation," Science, vol. 267, no. 5198, pp. 661-663, 1995.

[45] http://www.fei.com/products/transmission-electron-microscopes/titan/etem.aspx.

[46] http://www.jeolusa.com/PRODUCTS/ElectronOptics/TransmissionElectronMicroscopesTEM/Software/PracticalRemoteInSituMicroscopyPRISM/tabid/596/Default.aspx.

[47] P. L. Gai and E. D. Boyes, "Angstrom analysis with dynamic in situ aberration corrected electron microscopy," Journal of Physics: Conference Series, vol. 241, no. 1, Article ID 012055, 2010.

[48] R. M. Tromp and M. C. Reuter, "Design of a new photoemission/low-energy electron microscope for surface studies," Ultramicrosccopy, vol. 36, pp. 99-106, 1991.

[49] A. Botman, J. J. L. Mulders, R. Weemaes, and S. Mentink, "Purification of platinum and gold structures after electronbeam-induced deposition," Nanotechnology, vol. 17, no. 15, pp. 3779-3785, 2006.

[50] http://www.protochips.com/products/aduro.html.

[51] M. I. van der Meulen, N. Petkov, M. A. Morris et al., "Single crystalline $\mathrm{Ge}_{1-x} \mathrm{Mn}_{x}$ nanowires as building blocks for nanoelectronics," Nano Letters, vol. 9, no. 1, pp. 50-56, 2009.

[52] C. T. Harris, J. A. Martinez, E. A. Shaner et al., "Fabrication of a nanostructure thermal property measurement platform," Nanotechnology, vol. 22, no. 27, Article ID 275308, 2011.

[53] A. Lei, D. H. Petersen, T. J. Booth et al., "Customizable in situ TEM devices fabricated in freestanding membranes by focused ion beam milling," Nanotechnology, vol. 21, no. 40, Article ID 405304, 2010.

[54] K. Mølhave, S. B. Gudnason, A. T. Pedersen, C. H. Clausen, A. Horsewell, and P. Bøggild, "Transmission electron microscopy study of individual carbon nanotube breakdown caused by joule heating in air," Nano Letters, vol. 6, no. 8, pp. 1663-1668, 2006.

[55] K. H. Baloch, N. Voskanian, M. Bronsgeest, and J. Cumings, "Remote Joule heating by a carbon nanotube," Nature Nanotechnology, vol. 7, pp. 316-319, 2012.

[56] M. Hummelgård, R. Zhang, T. Carlberg et al., "Nanowire transformation and annealing by Joule heating," Nanotechnology, vol. 21, no. 16, Article ID 165704, 2010.

[57] H. Kohno, Y. Mori, S. Takeda, Y. Ohno, I. Yonenaga, and S. Ichikawa, "In situ transmission electron microscopy observation of the graphitization of silicon carbide nanowires induced by joule heating," Applied Physics Express, vol. 3, no. 5, Article ID 055001, 2010.

[58] L. T. Ngo, D. Almécija, J. E. Sader et al., "Ultimate-strength germanium nanowires," Nano Letters, vol. 6, pp. 2964-2968, 2006.

[59] B. Peng, M. Locascio, P. Zapol et al., "Measurements of near-ultimate strength for multiwalled carbon nanotubes and irradiation-induced crosslinking improvements," Nature Nanotechnology, vol. 3, no. 10, pp. 626-631, 2008.
[60] R. A. Bernal, R. Agrawal, B. Peng et al., "Effect of growth orientation and diameter on the elasticity of GaN nanowires. A combined in situ TEM and atomistic modeling investigation," Nano Letters, vol. 11, no. 2, pp. 548-555, 2011.

[61] H. Guo, K. Chen, Y. Oh et al., "Mechanics and dynamics of the strain-induced M1-M2 structural phase transition in individual $\mathrm{VO}_{2}$ nanowires," Nano Letters, vol. 11, pp. 3207-3213, 2011.

[62] Z. L. Wang, Z. R. Dai, R. Gao, and J. L. Gole, "Measuring the Young's modulus of solid nanowires by in situ TEM," Journal of Electron Microscopy, vol. 51, pp. S79-S85, 2002.

[63] D.-M. Tang, C.-L. Ren, X. Wei et al., "Mechanical properties of bamboo-like boron nitride nanotubes by in situ TEM and MD simulations: strengthening effect of interlocked joint interfaces," ACS Nano, vol. 5, pp. 7362-7368, 2011.

[64] Y. Lu, C. Peng, Y. Ganesan, J. Y. Huang, and J. Lou, "Quantitative in situ TEM tensile testing of an individual nickel nanowire," Nanotechnology, vol. 22, no. 35, Article ID 355702, 2011.

[65] E. Ruska, "Beitrag zur übermikroskopischen Abbildung bei höheren Drucken," Kolloid-Zeitschrift, vol. 100, no. 2, pp. 212219, 1942.

[66] M. J. Williamson, R. M. Tromp, P. M. Vereecken, R. Hull, and F. $\mathrm{M}$. Ross, "Dynamic microscopy of nanoscale cluster growth at the solid-liquid interface," Nature Materials, vol. 2, pp. 532-536, 2003.

[67] K.-L. Liu, C.-C. Wu, Y.-J. Huang et al., "Novel microchip for in situ TEM imaging of living organisms and bio-reactions in aqueous conditions," LAb on a Chip, vol. 8, pp. 1915-1921, 2008.

[68] N. Kolmakova and A. Kolmakov, "Scanning electron microscopy for in situ monitoring of semiconductor-liquid interfacial processes: electron assisted reduction of $\mathrm{Ag}$ ions from aqueous solution on the surface of $\mathrm{TiO}_{2}$ rutile nanowire," Journal of Physical Chemistry C, vol. 114, no. 40, pp. 17233-17237, 2010.

[69] Y. Xia, P. Yang, Y. Sun et al., "One-dimensional nanostructures: synthesis, characterization, and applications," Advanced Materials, vol. 15, no. 5, pp. 353-389, 2003.

[70] S. Helveg, C. López-Cartes, J. Sehested et al., "Atomic-scale imaging of carbon nanofibre growth," Nature, vol. 427, no. 6973, pp. 426-429, 2004.

[71] R. Sharma, P. Rez, M. Brown, G. Du, and M. M. J. Treacy, "Dynamic observations of the effect of pressure and temperature conditions on the selective synthesis of carbon nanotubes," Nanotechnology, vol. 18, no. 12, Article ID 125602, 2007.

[72] M. Lin, J. P. Y. Tan, C. Boothroyd, K. P. Loh, E. S. Tok, and Y. L. Foo, "Dynamical observation of bamboo-like carbon nanotube growth," Nano Letters, vol. 7, no. 8, pp. 2234-2238, 2007.

[73] C. Kallesøe, C.-Y. Wen, K. Mølhave, P. Bøggild, and F. M. Ross, "Measurement of local Si-nanowire growth kinetics using in situ transmission electron microscopy of heated cantilevers," Small, vol. 6, pp. 2058-2064, 2010.

[74] J. B. Hannon, S. Kodambaka, F. M. Ross, and R. M. Tromp, "The influence of the surface migration of gold on the growth of silicon nanowires," Nature, vol. 440, no. 7080, pp. 69-71, 2006.

[75] F. M. Ross, J. Tersoff, and M. C. Reuter, "Sawtooth faceting in silicon nanowires," Physical Review Letters, vol. 95, Article ID 146104, 4 pages, 2005.

[76] J. E. Allen, E. R. Hemesath, D. E. Perea et al., "High-resolution detection of Au catalyst atoms in Si nanowires," Nature Nanotechnology, vol. 3, no. 3, pp. 168-173, 2008.

[77] E. Sutter and P. Sutter, "Phase diagram of nanoscale alloy particles used for vapor-liquid-solid growth of semiconductor nanowires," Nano Letters, vol. 8, no. 2, pp. 411-414, 2008. 
[78] A. D. Gamalski, J. Tersoff, R. Sharma, C. Ducati, and S. Hofmann, "Formation of metastable liquid catalyst during subeutectic growth of germanium nanowires," Nano Letters, vol. 10, no. 8, pp. 2972-2976, 2010.

[79] A. D. Gamalski, C. Ducati, and S. Hofmann, "Cyclic supersaturation and triple phase boundary dynamics in germanium nanowire growth," Journal of Physical Chemistry C, vol. 115, no. 11, pp. 4413-4417, 2011.

[80] C. B. Collins, R. O. Carlson, and C. J. Gallagher, "Properties of gold-doped silicon," Physical Review, vol. 105, p. 1168, 1957.

[81] E. Koren, G. Elias, A. Boag, E. R. Hemesath, L. J. Lauhon, and Y. Rosenwaks, "Direct measurement of individual deep traps in single silicon nanowires," Nano Letters, vol. 11, no. 6, pp. 24992502, 2011.

[82] B. S. Kim, T. W. Koo, J. H. Lee et al., "Catalyst-free growth of single-crystal silicon and germanium nanowires," Nano Letters, vol. 9, no. 2, pp. 864-869, 2009.

[83] R. G. Hobbs, S. Barth, N. Petkov et al., "Seedless growth of sub-10 nm germanium nanowires," Journal of the American Chemical Society, vol. 132, no. 39, pp. 13742-13749, 2010.

[84] D. Li, M. H. Nielsen, J. R. I. Lee, C. Frandsen, J. F. Banfield, and J. J. De Yoreo, "Direction-specific interactions control crystal growth by oriented attachment," Science, vol. 336, pp. 1014-1018, 2012.

[85] H. G. Liao, L. Cui, S. Whitelam, and H. Zheng, "Real-time imaging of $\mathrm{Pt}_{3} \mathrm{Fe}$ nanorod growth in solution," Science, vol. 336, pp. 1011-1014, 2012.

[86] F. Léonard and A. A. Talin, "Electrical contacts to one- and twodimensional nanomaterials," Nature Nanotechnology, vol. 6, pp. 773-783, 2011.

[87] W. Lu, P. Xie, and C. M. Lieber, "Nanowire transistor performance limits and applications," IEEE Transactions on Electron Devices, vol. 55, no. 11, pp. 2859-2876, 2008.

[88] I. Ferain, C. A. Colinge, and J. P. Colinge, "Multigate transistors as the future of classical metal-oxide-semiconductor field-effect transistors," Nature, vol. 479, pp. 310-316, 2012.

[89] K. C. Lu, K. N. Tua, W. W. Wu, L. J. Chen, B. Y. Yoo, and N. V. Myung, "Point contact reactions between $\mathrm{Ni}$ and Si nanowires and reactive epitaxial growth of axial nano-NiSi/Si," Applied Physics Letters, vol. 90, Article ID 253111, 3 pages, 2007.

[90] W. W. Wu, K. C. Lu, C. W. Wang et al., "Growth of multiple metal/semiconductor nanoheterostructures through point and line contact reactions," Nano Letters, vol. 10, no. 10, pp. 39843989, 2010.

[91] Y. C. Chou, W. W. Wu, C. Y. Lee, C. Y. Liu, L. J. Chen, and K. N. $\mathrm{Tu}$, "Heterogeneous and homogeneous nucleation of epitaxial $\mathrm{NiSi}_{2}$ in [110] Si nanowires," Journal of Physical Chemisty C, vol. 115, no. 2, pp. 397-401, 2011.

[92] Y. C. Chou, W. W. Wu, S. L. Cheng et al., "In situ TEM observation of repeating events of nucleation in epitaxial growth of nano $\mathrm{CoSi}_{2}$ in nanowires of $\mathrm{Si}$," Nano Letters, vol. 8, no. 8, pp. 2194-2199, 2008.

[93] K. C. Lu, W. W. Wu, H. Ouyang et al., "The influence of surface oxide on the growth of metal/semiconductor nanowires," Nano Letters, vol. 11, pp. 2753-2758, 2011.

[94] J. Tang, C. Y. Wang, F. Xiu et al., "Single-crystalline $\mathrm{Ni}_{2} \mathrm{Ge} / \mathrm{Ge} /$ $\mathrm{Ni}_{2} \mathrm{Ge}$ nanowire heterostructure transistors," Nanotechnology, vol. 21, no. 50, Article ID 505704, 2010.

[95] T. Burchhart, A. Lugstein, Y. J. Hyun, G. Hochleitner, and E. Bertagnolli, "Atomic scale alignment of copper-germanide contacts for ge nanowire metal oxide field effect transistors," Nano Letters, vol. 9, no. 11, pp. 3739-3742, 2009.
[96] L. J. Lauhon, M. S. Gudikse, D. Wang, and C. M. Lieber, "Epitaxial core-shell and core-multishell nanowire heterostructures," Nature, vol. 40, pp. 57-61, 2002.

[97] S. Hu, Y. Kawamura, K. C. Y. Huang et al., “Thermal stability and surface passivation of Ge nanowires coated by epitaxial SiGe shells," Nano Letters, vol. 12, pp. 1385-1391, 2012.

[98] E. Tutuc, J. Appenzeller, M. C. Reuter, and S. Guha, "Realization of a linear germanium nanowire p-n junction," Nano Letters, vol. 6, no. 9, pp. 2070-2074, 2006.

[99] S. Hoffmann, J. Bauer, C. Ronning et al., "Axial p-n junctions realized in silicon nanowires by ion implantation," Nano Letters, vol. 9, no. 4, pp. 1341-1344, 2009.

[100] C. Zeiner, A. Lugstein, T. Burchhart et al., "Atypical selfactivation of Ga dopant for Ge nanowire devices," Nano Letters, vol. 11, pp. 3108-3112, 2011.

[101] R. Duffy, M. J. H. Van Dal, B. J. Pawlak et al., "Solid phase epitaxy versus random nucleation and growth in sub- $20 \mathrm{~nm}$ wide fin field-effect transistors," Applied Physics Letters, vol. 90, no. 24, Article ID 241912, 2007.

[102] R. Duffy, M. Shayesteh, B. McCarthy et al., "The curious case of thin-body Ge crystallization," Applied Physics Letters, vol. 99, no. 13, Article ID 131910, 3 pages, 2011.

[103] L. Pelaz, L. Marques, M. Aboy, P. Lopez, I. Santos, and R. Duffy, "Atomistic process modeling based on Kinetic Monte Carlo and Molecular Dynamics for optimization of advanced devices," in Proceedings of the IEEE International Electron Devices Meeting (IEDM '09), pp. 1-4, 2009, IEDM09-516, 97-4244-5640-6/09.

[104] N. Petkov, R. Kelly, M. Schmidt, and J. D. Holmes, "In situ dynamic TEM studies of Ga-ion implantation, subsequent defects evolution and thermal curing of Ge nanowires," in Proceedings of the European Microscopy Congress, vol. 10, PS1.10, 2012.

[105] D. J. Milliron, S. Raoux, R. M. Shelby, and J. Jordan-Sweet, "Solution-phase deposition and nanopatterning of GeSbSe phase-change materials," Nature Materials, vol. 6, no. 5, pp. 352356, 2007.

[106] J. W. L. Yim, B. Xiang, and J. Wu, "Sublimation of GeTe nanowires and evidence of its size effect studied by in situ TEM," in, Journal of the American Chemical Society, , vol. 131, pp. 1452614530, 2009.

[107] J. In, Y. Yoo, J. G. Kim et al., "In situ TEM observation of heterogeneous phase transition of a constrained singlecrystalline $\mathrm{Ag}_{2} \mathrm{Te}$ nanowire," Nano Letters, vol. 10, no. 11, pp. 4501-4504, 2010.

[108] X. Liu, J. Zhu, C. Jin, L.-M. Peng, D. Tang, and H. Cheng, "In situ electrical measurements of polytypic silver nanowires," Nanotechnology, vol. 19, Article ID 085711, 6 pages, 2008.

[109] Y. G. Wang, T. H. Wang, X. W. Lin, and V. P. Dravid, "Ohmic contact junction of carbon nanotubes fabricated by in situ electron beam deposition," Nanotechnology, vol. 17, no. 24, pp. 6011-6015, 2006.

[110] S. Kumar, K. L. Joshi, A. C. T. van Duin, and M. A. Haque, "Can amorphization take place in nanoscale interconnects?" Nanotechnology, vol. 23, no. 9, Article ID 095701, 2012.

[111] A. Reguer, F. Bedu, S. Nitsche, D. Chaudanson, B. Detailleur, and H. Dallaporta, "Probing the local temperature by in situ electron microscopy on a heated $\mathrm{Si}_{3} \mathrm{~N}_{4}$ membrane," Ultramicroscopy, vol. 110, no. 1, pp. 61-66, 2009.

[112] S. Chen, J. Y. Huang, Z. Wang, and K. Kempa, "High-biasinduced structure and the corresponding electronic property changes in carbon nanotubes," Applied Physics Letters, vol. 87, Article ID 263107, 2005. 
[113] C. H. Jin, J. Y. Wang, Q. Chen, and L. M. Peng, "In situ fabrication and graphitization of amorphous carbon nanowires and their electrical properties," Journal of Physical Chemistry B, vol. 110, no. 11, pp. 5423-5428, 2006.

[114] B. Kang and G. Ceder, "Battery materials for ultrafast charging and discharging," Nature, vol. 458, no. 7235, pp. 190-193, 2009.

[115] C. K. Chan, H. Peng, G. Liu et al., "High-performance lithium battery anodes using silicon nanowires," Nature Nanotechnology, vol. 3, no. 1, pp. 31-35, 2008.

[116] K. Karki, E. Epstein, J.-H. Cho et al., "Lithium-assisted electrochemical welding in silicon nanowire battery electrodes," Nano Letters, vol. 12, pp. 1392-1397, 2012.

[117] X. H. Liu, S. Huang, S. T. Picraux, J. Li, T. Zhu, and J. Y. Huang, "Reversible nanopore formation in Ge nanowires during lithiation-delithiation cycling: an in situ transmission electron microscopy study," Nano Letters, vol. 11, pp. 3991-3997, 2011.

[118] X. Wang, D.-M. Tang, H. Li et al., "Revealing the conversion mechanism of $\mathrm{CuO}$ nanowires during lithiation-delithiation by in situ transmission electron microscopy," Chemical Communications, vol. 48, pp. 4812-4814, 2012.

[119] Y. Liu, N. S. Hudak, D. L. Huber, S. J. Limmer, J. P. Sullivan, and J. Y. Huang, "In situ transmission electron microscopy observation of pulverization of aluminum nanowires and evolution of the thin surface $\mathrm{Al}_{2} \mathrm{O}_{3}$ layers during lithiation-delithiation cycles," Nano Letters, vol. 11, pp. 4188-4194, 2011.

[120] P. Gao, K. Liu, L. Liu et al., "Higher-order harmonic resonances and mechanical properties of individual cadmium sulphide nanowires measured by in situ transmission electron microscopy," Journal of Electron Microscopy, vol. 59, no. 4, pp. 285-289, 2010.

[121] B. Liu, Y. Bando, M. Wang, C. Tang, M. Mitome, and D. Golberg, "Crystallography and elasticity of individual GaN nanotubes," Nanotechnology, vol. 20, no. 18, Article ID 185705, 2009.

[122] Y. Yue, P. Liu, Z. Zhang, X. Han, and E. Ma, "Approaching the theoretical elastic strain limit in copper nanowires," Nano Letters, vol. 11, pp. 3151-3155, 2011. 

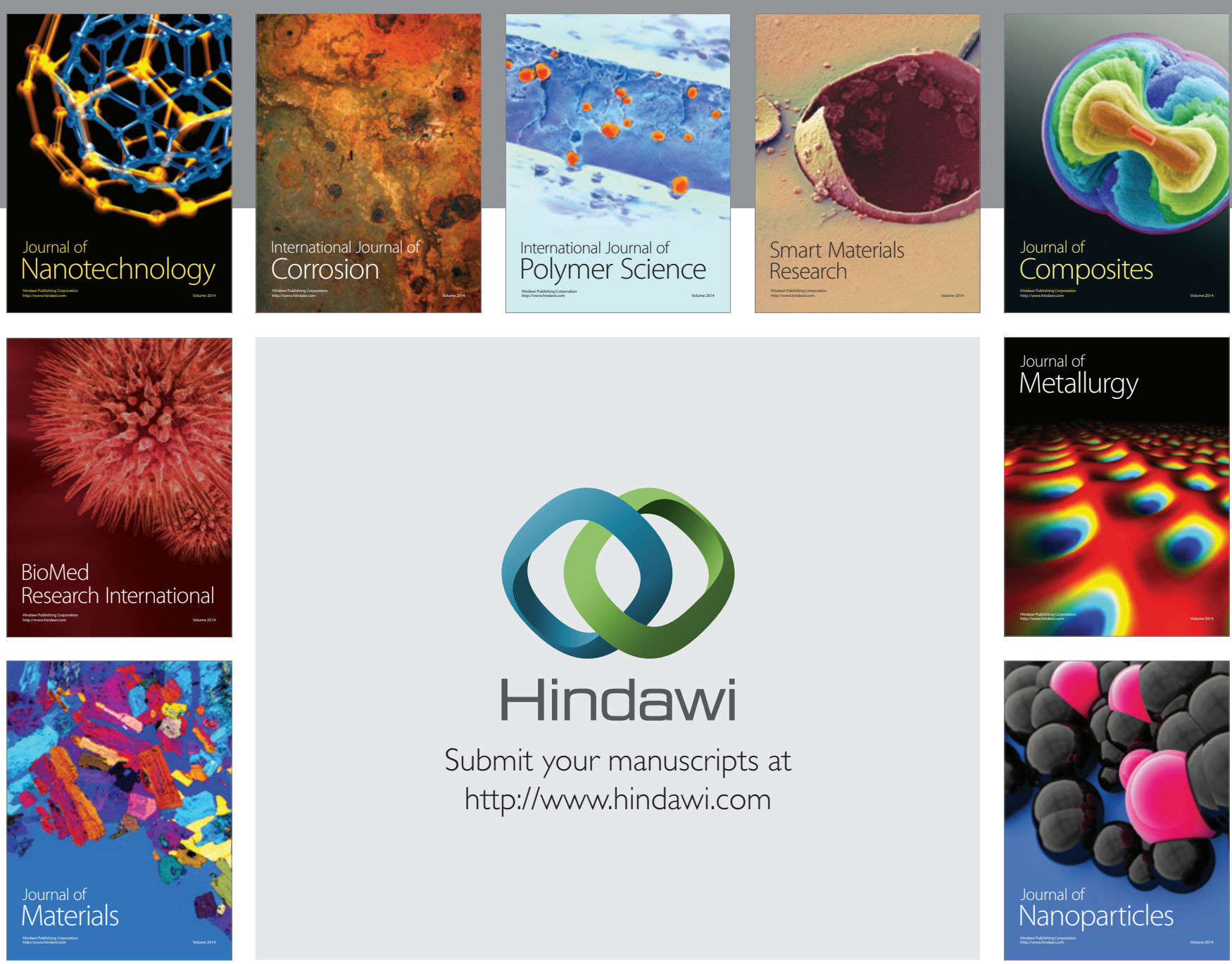

Submit your manuscripts at http://www.hindawi.com
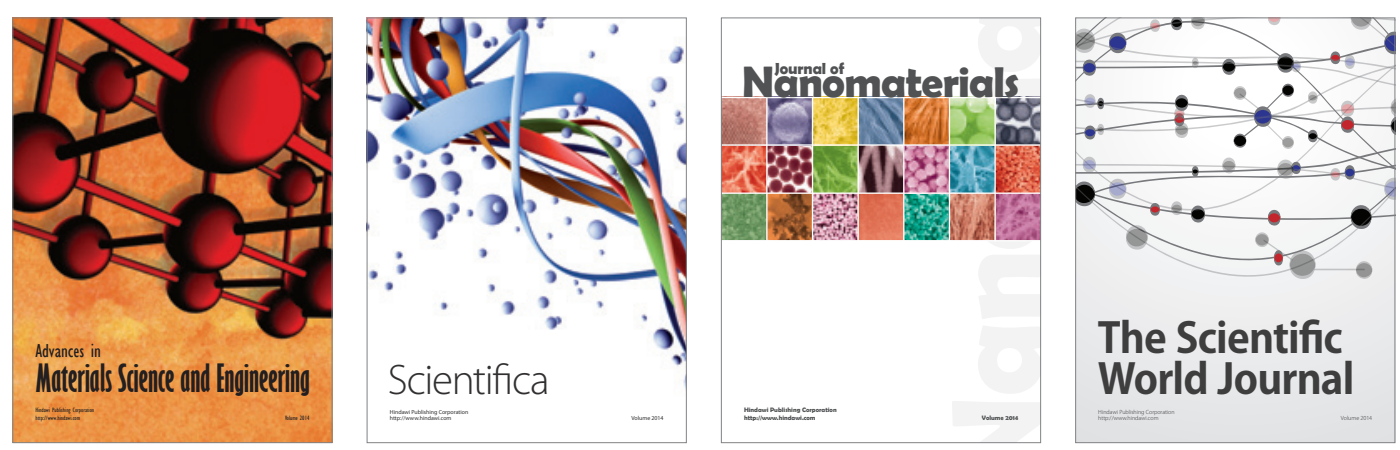

\section{The Scientific World Journal}
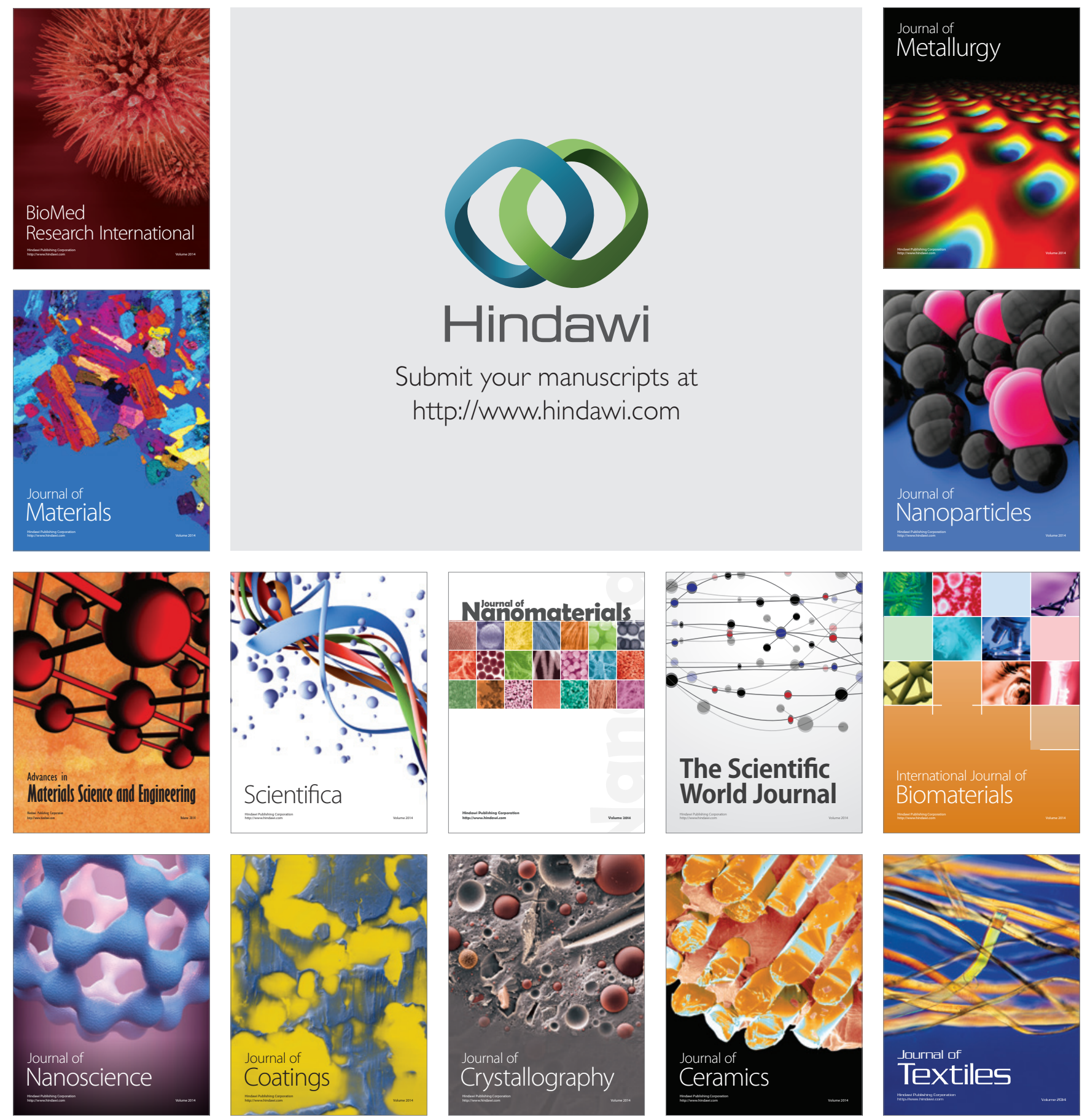